\title{
Approximation properties of receding horizon optimal control
}

\section{Lars Grüne}

\begin{abstract}
In this survey, receding horizon control is presented as a method for obtaining approximately optimal solutions to infinite horizon optimal control problems by iteratively solving a sequence of finite horizon optimal control problems. We investigate conditions under which we can obtain mathematically rigorous approximation results for this approach. A key ingredient of our analysis is the so-called turnpike property of optimal control problems.
\end{abstract}

Keywords Optimal control; receding horizon control; model predictive control; turnpike property; dissipativity

\section{Introduction}

Receding horizon control (RHC) is a method for constructing a control function on an arbitrary long time horizon from the iterative solution of finite horizon optimal control problems. Although particularly popular in control engineering - where it usually comes under the name Model Predictive Control (MPC), see, e.g., [21,28,38,46] — conceptually similar ideas have been used also in mathematical economy under the name sliding plan [34] or in operations research under the name rolling horizon [10].

In the context of this paper, RHC is seen as a method for the approximate solution of optimal control problems on an infinite time horizon. Of course, in real life applications infinite time horizons do not exist, as everything will

L. Grüne

Mathematical Institute

University of Bayreuth

95440 Bayreuth, Germany

E-mail: lars.gruene@uni-bayreuth.de

The paper was written while the author was visiting the University of Newcastle, Australia. The author is grateful to Arthur Fleig for providing the numerical simulations for Example 3.3 and to Arthur Fleig, Chris Kellett and Steve Weller for very helpful comments on a draft of this paper. 
eventually come to an end. Nevertheless, optimal control problems on infinite time horizons are practically relevant as they are always the method of choice when systems need to be run in an efficient way for indefinitely long time spans. This includes optimal stabilisation and tracking problems (e.g., keeping the temperature of a building within a given range with minimal energy consumption), optimal process control (e.g., running a continuous chemical reactor at an optimal operating point) but also economic problems like maximising utility in a macroeconomical model [12].

The key idea of RHC, first presented in the 1960s [44], lies in synthesising a control strategy on an arbitrary long time horizon by glueing together pieces of finite horizon optimal control problems. Since the finite horizon optimal control problems are solved iteratively based on the most recent information, the receding horizon control method provides the optimal control in the form of a feedback law, which provides a certain robustness against perturbation and model uncertainty. This aspect is often seen as the main feature of RHC. In this paper, however, we want to highlight another essential property of RHC, namely the fact that finite horizon optimal control problems are often much easier to solve than infinite horizon problems, which implies that RHC provides a way to make optimal control problems computationally tractable which could not be solved otherwise. From this point of view, RHC may be seen as a model reduction technique in time, in which the solution of a difficult problem (the infinite horizon optimal control problem) is replaced by a sequence of solutions of easier problems (the finite horizon problems). Like other model reduction techniques, it relies on the fact that the full problem contains a sufficient amount of redundancy, which can be exploited for simplifying the problem. This survey article presents a number of recent results from this area, in which the redundancy stems from the fact that the optimal trajectories "most of the time" stay close to an optimal equilibrium. This property, known in the optimal control literature as the turnpike property, will play a key role in our analysis. Interestingly, the turnpike property, which is a classical property in optimal control $[52,18,39,14]$ has recently attracted renewed attention, not least because of its importance for Receding Horizon Control [16, 19, 20,50, 42].

Receding Horizon or Model Predictive Control has been a topic of intensive research in control engineering for more than four decades. However, the point of view on the method we take in this survey has a different focus. While control engineers primarily see MPC as an online control algorithm, in which optimality may well be compromised by other important objectives like realtime capability, efficient handling of constraints or robustness against certain classes of perturbations, optimality is the focus of this paper. More precisely, we ask the question: under which conditions is it possible, if at all, to approximate the optimal solution of an infinite horizon problem by solving a sequence of finite horizon problems? This is also why in this context we prefer the name "receding horizon control", because although in the literature the names "receding horizon control" and "model predictive control" are largely used synonymously, the latter is often regarded as an online control scheme 
while the former rather denotes a particular way of approaching optimal control problems, which is more in line with the focus of this survey paper. In this context it should be noted that the majority of results in this paper apply to what in the MPC community is called Economic $M P C[5,6,17,25,32]$. The "classical" MPC setting in which the cost function is supposed to be positive definite is addressed in Section 7 of this paper.

Regarding its contribution, this paper is in fact somewhat more than a survey, because while the results presented here have already appeared in the literature, the way the basic conditions in Sections 4 and 5 are written and the way that the proofs are organised is new and has not been presented before elsewhere. The paper is organised as follows. After defining the setting and the problem formulation in Section 2, we demonstrate the performance of RHC with three examples in Section 3. These examples will also be used in order to illustrate the methods and results introduced later in the paper. Section 4 defines the turnpike properties used in this paper and states and proves the first approximation result. Section 5 shows how the assumptions from Section 4 can be guaranteed by means of dissipativity and controllability notions, which are easier to check for concrete examples. Section 6 explains how terminal conditions can be used in order to improve the performance of the method and Section 7 addresses problems with positive definite stage cost, which are related to stabilisation and tracking problems.

\section{Setting and preliminaries}

We consider nonlinear discrete time control systems

$$
x(k+1)=f(x(k), u(k))
$$

for $f: X \times U \rightarrow X$, with normed spaces $X$ and $U$ denoting the state and control space, respectively. The solution of system (2.1) for a control sequence $u=$ $(u(0), u(1), \ldots, u(K-1)) \in U^{K}$ emanating from the initial value $x$ is denoted by $x_{u}(k, x), k=0, \ldots, K-1$. The set $\mathbb{Y} \subset X \times U$ denotes the admissible state-control pairs, i.e., the combined state/control constraints and $\mathbb{X}:=\{x \in$ $X \mid$ there exists $u \in U$ with $(x, u) \in \mathbb{Y}\}$ and $\mathbb{U}(x):=\{u \in U \mid(x, u) \in \mathbb{Y}\}$ denote the induced sets of admissible states and controls. For a given initial value $x \in \mathbb{X}$, a control sequence $u \in U^{K}$ is called admissible if $\left(x_{u}(k, x), u(k)\right) \in$ $\mathbb{Y}$ holds for all time instants $k=0, \ldots, K-1$ and $x_{u}(K, x) \in \mathbb{X}$. The set of all admissible control sequences is denoted by $\mathbb{U}^{K}(x)$. For the infinite horizon case with $u=(u(0), u(1), \ldots) \in U^{\infty}$ we define the set $\mathbb{U}^{\infty}(x)$ analogously. As the focus in this paper is on optimality properties rather than on constraints, in order to keep the presentation technically simple we assume that $\mathbb{U}^{\infty}(x) \neq \emptyset$ for all $x \in \mathbb{X}$.

In practice, control systems are often defined in continuous time with dynamics governed by ordinary or partial differential equations rather than by discrete time dynamics of the type (2.1). However, choosing $f$ in (2.1) as the solution operator of a controlled ordinary or partial differential equation over 
a sampling interval $\left[0, T_{s}\right]$ for sampling time $T_{s}>0$, we can always convert continuous time models into discrete time models of the type (2.1), see also Example 3.3, below. Alternatively, one can formulate receding horizon control schemes directly in continuous time and we will provide corresponding references to the literature at the end of each section. Since the discrete time formulation avoids a number of technical difficulties, we decided to adopt this variant in this paper.

Receding horizon control in the sense of this paper is an approximate solution approach for infinite horizon optimal control problems by solving a sequence of finite horizon optimal control problems. These problems are defined as follows.

For a given stage cost $\ell: \mathbb{Y} \rightarrow \mathbb{R}$, we define the finite horizon functional

$$
J_{N}(x, u):=\sum_{k=0}^{N-1} \ell\left(x_{u}(k, x), u(k)\right)
$$

and the corresponding infinite horizon functional

$$
J_{\infty}(x, u):=\limsup _{N \rightarrow \infty} J_{N}(x, u) .
$$

The optimal control problems under consideration now consist in minimising the objective with respect to the control sequence $u$, i.e., to solve the problems

$$
\underset{u \in \mathbb{U}^{N}(x)}{\operatorname{minimise}} J_{N}(x, u)
$$

or, respectively,

$$
\underset{u \in \mathbb{U} \infty(x)}{\operatorname{minimise}} J_{\infty}(x, u)
$$

for given initial condition $x \in \mathbb{X}$. More precisely, we are going to focus on the solution of the infinite horizon problem (2.5) and will investigate how this can approximately be achieved by iteratively solving finite horizon problems of the type (2.4).

Associated to the optimal control problems (2.4), (2.5) we define the optimal value functions

$$
V_{N}(x):=\inf _{u \in \mathbb{U}^{N}(x)} J_{N}(u, x) \quad \text { and } \quad V_{\infty}(x):=\inf _{u \in \mathbb{U}^{\infty}(x)} J_{\infty}(u, x) .
$$

The indexing of the sum in the finite horizon functional (2.2) from 0 to $N-1$ is a convention frequently found in RHC, since it permits the incorporation of additional terminal costs as the term with index $N$, see Section 6 . Note that this numbering implies that, without terminal costs, $N=2$ is the shortest meaningful horizon, because for $N=1$ the objective $J_{N}(x, u)$ is independent of the control $u$.

In the sequel we assume that for all $N \in \mathbb{N} \cup\{\infty\}$ and $x \in \mathbb{X}$ there is an optimal control sequence $u_{N, x}^{*} \in \mathbb{U}^{N}(x)$, i.e., a control sequence for which the equality $V_{N}(x)=J_{N}\left(x, u_{N, x}^{*}\right)$ holds. This assumption is mainly made 
for simplifying the presentation, as most statements could be rewritten using approximately optimal control sequences. We remark that optimal control sequences need not be unique; in this case $u_{N, x}^{*}$ denotes one of the possible optimal control sequences. We drop the index $x$ of $u_{N, x}^{*}$ whenever the initial condition is clear from the context.

We note that for the infinite horizon optimal (or approximately optimal) trajectory, for initial value $x$ to be well defined we need to ensure that the optimal value $V_{\infty}(x)$ is finite (i.e., neither $+\infty$ nor $-\infty$ ), which can be ensured, e.g., by establishing upper and lower bounds on $J_{\infty}(x, u)$. In the sequel, we will assume finiteness of $V_{\infty}(x)$ for all $x \in \mathbb{X}$ whenever we consider the infinite horizon problem.

Using the optimal control problem (2.2), (2.4), we now define the receding horizon control $(\mathrm{RHC})$ scheme we analyse in this paper.

Algorithm 2.1 Given an initial condition $x(0) \in \mathbb{X}$ and an optimisation horizon $N \in \mathbb{N}$, for $n=0,1,2, \ldots$ we perform the following steps:

1. Let $x=x(n)$ denote the current state of the system.

2. Solve the finite horizon optimal control problem (2.4) in order to obtain the optimal control sequence $u_{N, x}^{*}$.

3. Apply the first element of the optimal control sequence $u_{N, x}^{*}$ as a feedback control value until the next time instant, i.e., define the feedback law $\mu_{N}(x):=u_{N, x}^{*}(0)$ and set $x(n+1):=f\left(x(n), \mu_{N}(x(n))\right)$.

4. Set $n:=n+1$ and go to Step 1 .

The system $x(n+1)=f\left(x(n), \mu_{N}(x(n))\right)$ is referred to as the RHC closed loop system. Trajectories of this system with initial value $x \in \mathbb{X}$ will be denoted by $x_{\mu_{N}}(n, x)$ and referred to as RHC solutions or RHC trajectories.

As already explained in the Introduction, the focus of this paper will be on analysing the optimality properties of the RHC solutions. To this end, we define the closed loop performance criterion

$$
J_{M}^{c l}\left(x, \mu_{N}\right):=\sum_{n=0}^{M-1} \ell\left(x_{\mu_{N}}(n, x), \mu_{N}\left(x_{\mu_{N}}(n, x)\right)\right)
$$

for arbitrary $M \in \mathbb{N}$. If the performance value is bounded as a function in $M$, we also consider the infinite horizon performance measure

$$
J_{\infty}^{c l}\left(x, \mu_{N}\right):=\limsup _{M \rightarrow \infty} J_{M}^{c l}\left(x, \mu_{N}\right) .
$$

We emphasise that these performance criteria yield tighter notions of optimality than the averaged value $\lim \sup _{M \rightarrow \infty} \frac{1}{M} J_{M}^{c l}\left(x, \mu_{N}\right)$ which is often studied in the $\mathrm{RHC} / \mathrm{MPC}$ literature, e.g., in $[5,6]$, because in the averaged functional the behaviour of $x_{\mu_{N}}$ on any finite time interval effectively plays no role. 
Throughout the paper we will make use of the following classes of comparison functions

$$
\begin{aligned}
& \mathcal{L}:=\left\{\begin{array}{l|l}
\delta: \mathbb{R}_{0}^{+} \rightarrow \mathbb{R}_{0}^{+} & \begin{array}{l}
\delta \text { continuous and decreasing } \\
\text { with } \lim _{k \rightarrow \infty} \delta(k)=0
\end{array}
\end{array}\right\}, \\
& \mathcal{K}:=\left\{\begin{array}{l|l}
\alpha: \mathbb{R}_{0}^{+} \rightarrow \mathbb{R}_{0}^{+} & \begin{array}{l}
\alpha \text { continuous and strictly } \\
\text { increasing with } \alpha(0)=0
\end{array}
\end{array}\right\}, \\
& \mathcal{K}_{\infty}:=\{\alpha \in \mathcal{K} \mid \alpha \text { unbounded }\} .
\end{aligned}
$$

Moreover, we will use the dynamic programming principles for $V_{N}$ and $V_{\infty}$ which for all $K=1, \ldots, N-1$ or all $K \in \mathbb{N}$, respectively, read

$$
\begin{aligned}
V_{\infty}(x) & =\inf _{u \in \mathbb{U}^{K}(x)}\left\{J_{K}(x, u)+V_{\infty}\left(x_{u}(K, x)\right)\right\} \\
& = \\
V_{N}(x) & =\inf _{u \in \mathbb{U}^{K}(x)}\left\{\left(x, u_{\infty, x}^{*}\right)+V_{\infty}\left(x_{u_{\infty, x}^{*}}(K, x)\right)\right. \\
& \left.=\quad J_{K}(x, u)+V_{N-K}\left(x_{u}(K, x)\right)\right\}
\end{aligned}
$$

cf. [28, Theorem 3.15 and Theorem 4.4]. We remark that this principle implies that tails of optimal trajectories are again optimal trajectories, i.e., that the identity

$$
J_{N-K}\left(x_{u_{N, x}^{*}}(k, x), u_{N, x}^{*}(\cdot+K)\right)=V_{N-K}(x)
$$

holds, see [28, Corollary 3.16]

\section{Examples}

Before presenting the theoretical analysis of the RHC method, in this section we present three examples which will later be used in order to illustrate the methods and results we present in this paper. Two of the examples are one dimensional discrete time systems which were selected because, due their simplicity, we can provide all computations needed in our analysis in detail. The third example is an optimal control problem for a parabolic PDE, presented in order to demonstrate that both the RHC method and our analysis are also applicable to more complex examples.

Example 3.1 Consider the control system

$$
x(k+1)=2 x(k)+u(k)
$$

with $X=U=\mathbb{R}$ and $\mathbb{Y}=\mathbb{X} \times \mathbb{U}=[-0.5,0.5] \times[-2,2]$. The stage cost $\ell$ is chosen such that the control effort is penalised quadratically, i.e.,

$$
\ell(x, u)=u^{2} .
$$

Hence, the optimal control problem tries to keep the system inside $\mathbb{X}$ with minimal control effort. It is easily seen that a good way of doing this is to steer 
the system to the equilibrium $x^{e}=0$, because at $x^{e}=0$ the cost for keeping the system inside $\mathbb{X}$ is $\ell(0,0)=0$.

Figure 3.1 shows the RHC trajectories $x(k)=x_{\mu_{N}}\left(k, x_{0}\right)$ for $x_{0}=0.5$ (solid) and the corresponding finite horizon optimal trajectories $x_{u_{N, x(k)}}(\cdot, x(k))$ for each step $k$ (dashed) for $N=5$ on the left and for $N=10$ on the right. One sees that while the finite horizon trajectories eventually move to the upper boundary of the admissible set $\mathbb{X}=[-0.5,0.5]$, the RHC trajectory tends towards a neighbourhood of $x^{e}=0$.
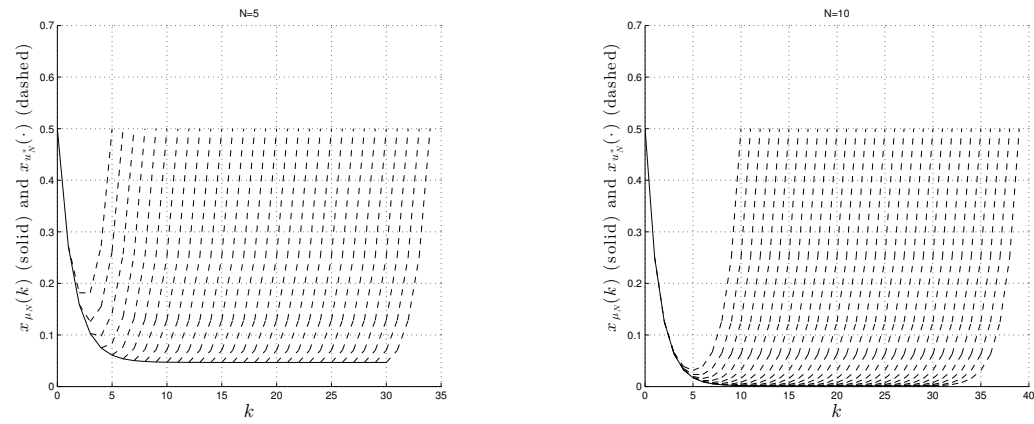

Fig. 3.1 RHC trajectory $x(k)=x_{\mu_{N}}\left(k, x_{0}\right)$ (solid) and finite horizon optimal trajectories $x_{u_{N, x(k)}^{*}}(\cdot, x(k))$ (dashed) along $x(k)$ for Example 3.1 with $x_{0}=0.5$ and $X=[-0.5,0.5]$ and optimisation horizon $N=5$ (left) and $N=10$ (right)

When increasing $N$, the RHC solution ends up in a smaller neighbourhood of $x^{e}$. This suggests that the cost of the RHC solutions should decrease with increasing $N$, which is what actually happens, as illustrated in Figure 3.2 for $J_{30}^{c l}\left(x, \mu_{N}\right)$.

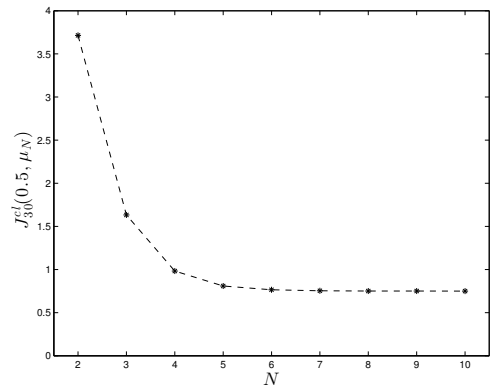

Fig. 3.2 $J_{30}^{c l}\left(x, \mu_{N}\right)$ for Example 3.1 with $N=2, \ldots, 10, x=0.5$ and $\mathbb{X}=[-0.5,0.5]$ 
Example 3.2 Our second example is a basic economic growth model which goes back to [12]. It is given by the dynamics and stage cost

$$
x(k+1)=u(k) \quad \text { and } \quad \ell(x, u)=-\ln \left(A x^{\alpha}-u\right),
$$

where $x$ denotes the capital stock, $A x^{\alpha}$ is a production function with constants $A>0,0<\alpha<1$, and the control variable $u$ determines the amount of capital to be kept in the next time step. The difference $A x^{\alpha}-u$ thus denotes the consumption whose logarithmic utility $\ln \left(A x^{\alpha}-u\right)$ is maximised. In order to be consistent with the general setting this has been reformulated as a minimisation problem for the negative logarithm.

Using the parameters $A=5$ and $\alpha=0.34$ and the constraints $\mathbb{Y}=\mathbb{X} \times \mathbb{U}$ with $\mathbb{X}=\mathbb{U}=[0.1,10]$ we have performed the same numerical simulations as in the previous example, see Figure 3.3.
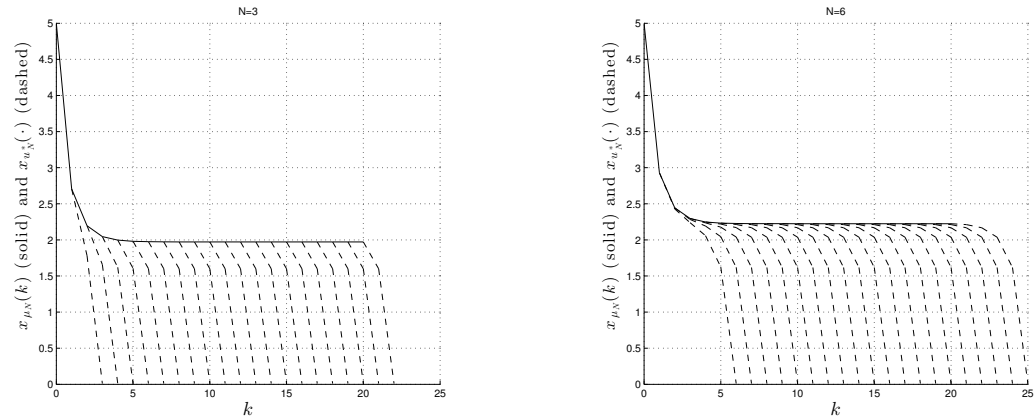

Fig. 3.3 RHC trajectory $x(k)=x_{\mu_{N}}\left(k, x_{0}\right)$ (solid) and finite horizon optimal trajectories $x_{u_{N, x(k)}^{*}}(\cdot, x(k))$ (dashed) along $x(k)$ for Example 3.2 with $x=5$ and optimisation horizon $N=5$ (left) and $N=10$ (right)

Except for the fact that now the finite horizon optimal trajectories end up at the lower boundary of $\mathbb{X}$, the graphs are qualitatively similar to those from Figure 3.1: while the finite horizon trajectories move to the boundary of the admissible $\mathbb{X}$, the RHC solutions converge to neighbourhoods of an equilibrium $x^{e}$ in its interior, whose sizes shrink with increasing $N$. A closer inspection reveals that this equilibrium is given by $x^{e}=\frac{1}{\sqrt[\alpha-1]{\alpha A}} \approx 2.234421144$, which happens to be the optimal equilibrium of the problem, i.e., the minimiser of $-\ln \left(A x^{\alpha}-u\right)$ under the constraints $x \in \mathbb{X}$ and $f(x, u)=u$ (which in this example is equivalent to $x=u)$.

The fact that the solutions end up closer to $x^{e}$ when $N$ increases suggests that the performance of the RHC trajectory should also improve with increasing $N$. Figure 3.4 shows that this is exactly what is happening here - just as for Example 3.1.

Example 3.3 Our third example is an application of RHC to the FokkerPlanck equation, first considered in [7,8]. The example differs from the first 


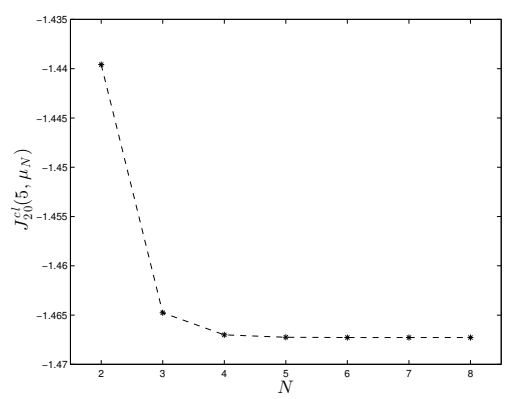

Fig. 3.4 $J_{20}^{c l}\left(x, \mu_{N}\right)$ for Example 3.2 with $N=2, \ldots, 8$ and $x=5$

two examples in two points: first, we are now considering a controlled PDE and second, we focus on the qualitative behaviour of the solutions.

In order to motivate the control of the Fokker-Planck equation, we consider the control of a large ensemble of identical stochastic systems, with the dynamics of each element in the ensemble governed by the controlled Itô stochastic differential equation (SDE)

$$
d X_{t}=b\left(X_{t}, t, u\right) d t+\sigma\left(X_{t}, t\right) d W_{t}
$$

with drift $b: \mathbb{R}^{d} \times \mathbb{R} \times \mathbb{R}^{m} \rightarrow \mathbb{R}^{d}$, diffusion $\sigma: \mathbb{R}^{d} \times \mathbb{R} \rightarrow \mathbb{R}^{d \times p}$, p-dimensional Wiener process $W_{t}$ and initial condition $X_{0} \in \mathbb{R}^{d}$. The distribution of a large ensemble is statistically determined by its time dependent probability density function (PDF) y: $\times \mathbb{R} \mathbb{R}^{d} \rightarrow \mathbb{R}_{0}^{+}$. The control task we are considering thus consists of controlling the PDF of the ensemble towards a desired reference density function $y_{\text {ref }}: \mathbb{R}^{d} \rightarrow \mathbb{R}_{0}^{+}$. Under suitable regularity conditions, the evolution of the PDF is determined by the Fokker-Planck partial differential equation

$$
\begin{aligned}
\partial_{t} y(t, x) & =\sum_{i, j=1}^{d} \partial_{i j}^{2}\left(a_{i j}(t, x) y(t, x)\right)+\sum_{i=1}^{d} \partial_{i}\left(b_{i}(t, x, u) y(t, x)\right) \\
y(0, x) & =y_{0}(x)
\end{aligned}
$$

for $(t, x) \in \mathbb{R}_{>0} \times \mathbb{R}^{d}$ and with $a_{i j}=\sum_{k} \sigma_{i k} \sigma_{j k} / 2$, for details see, e.g., [43, p. 227], [45, p. 297] or [48]. In (3.2), u can in principle be a function of time $t$ and/or state $x$. As in [7,8], here we focus on the case of u being independent of $x$. This means that when applying the control to (3.1) we do not need to know the current state $X_{t}$. In other words, each element of the ensemble receives the same control input, which makes the implementation of such a control particularly easy.

In order to apply the discrete time RHC framework described in the last section to this problem, we need to rewrite the sampled-data version of the Fokker-Planck equation as a discrete time system. To this end we fix a sampling 
time $T_{s}>0$, sampling instants $t_{n}:=n T_{s}$ for $n \in \mathbb{N}_{0}$ and the discrete time state

$$
z(n):=y\left(t_{n}, \cdot\right)
$$

which is now an element of an appropriate function space $X$, for details see [22]. Denoting the piece of the control function $u$ acting from $t_{n}$ to $t_{n+1}$ shifted to $\left[0, T_{s}\right]$ by $u(n)$ (i.e., $u(n)(t)=u\left(t+t_{n}\right)$ ) and denoting by $f$ the solution operator of the Fokker-Planck equation on the interval $\left[0, T_{s}\right]$, we can then write the discrete time dynamics as

$$
z(n+1)=f(z(n), u(n)), \quad z(0)=z_{0}=y_{0}
$$

(we use $z$ instead of $x$ now because $x$ already denotes the independent state variable in (3.2)). Note that $u(n)$ can be either time varying or constant in time on $\left[0, T_{s}\right]$; the latter setting was used in the numerical simulation presented below. We denote the space of admissible control inputs for $f$ by $U$. In order to make the optimal trajectories approach the desired reference PDF $y_{\text {ref }}$, we use the stage cost

$$
\ell(z, u)=\frac{1}{2}\left\|z-y_{r e f}\right\|_{L^{2}\left(\mathbb{R}^{d}\right)}^{2}+\frac{\nu}{2}\left\|u-u_{r e f}\right\|_{2}^{2},
$$

where $u_{\text {ref }} \in \mathbb{R}^{d}$ is a control value for which $y_{\text {ref }}$ becomes an equilibrium of (3.2) and $\nu>0$ is a weighting parameter.

In order to show the performance of $\mathrm{RHC}$ for this problem, as an example for (3.1) we consider a two-dimensional system consisting of two OrnsteinUhlenbeck processes. This amounts to choosing the diffusion as

$$
a_{i j}:=\delta_{i j} \sigma_{i}^{2} / 2, \quad i, j=1,2
$$

where $\sigma_{i}>0$, and $\delta_{i j}$ is the Kronecker delta. The drift is defined by

$$
b_{i}(t, x, u):=-\mu_{i} x_{i}+u_{i}, \quad i=1,2
$$

for $\mu_{i}>0$ and $u_{i} \in \mathbb{R}$. The parameters for the simulation are set to $\sigma_{i}=0.25$ and $\mu_{i}=1$ for $i=1$ and 2 . The sampling time is chosen as $T_{s}=0.1$ and the desired reference PDF is a 2d Gaussian distribution with mean $\mu=(3.5,3.5)^{T}$ and covariance matrix $\Sigma=\operatorname{diag}(\sqrt{2} / 8, \sqrt{2} / 8)$, which is an equilibrium of $(3.2)$ for $u_{\text {ref }}=(3.5,3.5)^{T}$. No state or control constraints were imposed.

We compute the RHC solution for the shortest meaningful optimisation horizon $N=2$. The initial PDF $z_{0}=y_{0}$ is again a 2d Gaussian with mean $\mu_{0}=(-3.5,-3.5)^{T}$ and covariance matrix $\Sigma=\operatorname{diag}(0.5,0.5)$. The weighting parameter is set to $\nu=0.25$. Figure 3.5 shows the reference PDF $y_{\text {ref }}$ at the right, the initial PDF $y_{0}$ on the left and the $R H C$ solution at time $t=$ 1 and $t=2$ in between. For the numerical discretisation the Chang-Cooper scheme in space and the BDF2 scheme in time was used [40] and the numerical optimisation was carried out using the necessary optimality conditions from [8] on the domain $\Omega=(-7,5)^{2}$.

The remarkable fact, which is consistent with the observations [7,8], is that even for the shortest meaningful optimisation horizon $N=2$ the trajectory 


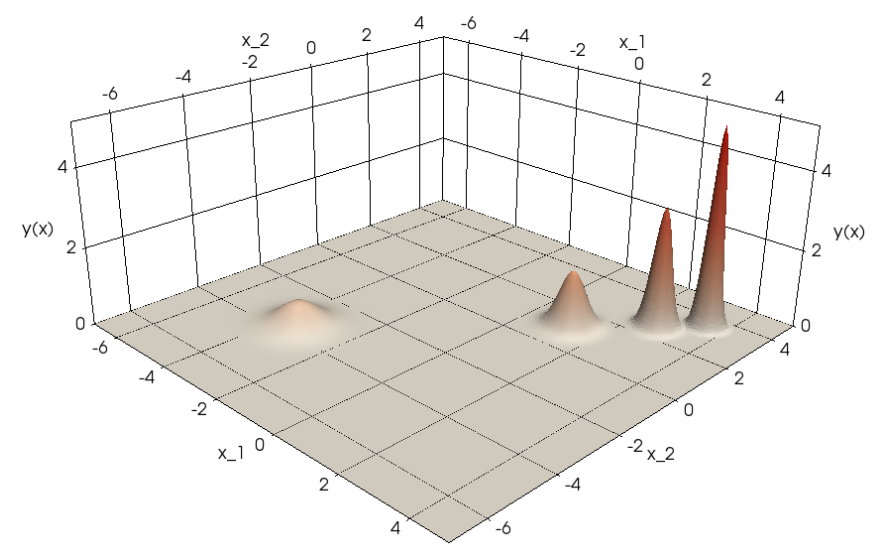

Fig. 3.5 RHC trajectory $z_{\mu_{N}}(n)=y\left(t_{n}, y_{0}\right)$ for $N=2$ at the three time instants $t_{n}=0,1,2$ (left to right) and reference PDF $y_{\text {ref }}$ (at the very right)

indeed converges to the desired reference PDF. We will see later in Example 7.5 that this is not a coincidence, but rather that this behaviour can actually be proved rigorously.

\section{Results for general stage cost}

As mentioned in the introduction, a reduction technique like RHC can only work if the optimal control problem has a suitable amount of redundancy. The kind of redundancy that we are assuming here is the so called turnpike property, which asserts that the optimal trajectories "most of the time" stay near an equilibrium $\left(x^{e}, u^{e}\right)$, cf. Part (i) and (ii) of the subsequent assumption. Here, the sets $\mathcal{Q}(x, P, \infty)$ and $\mathcal{Q}(x, P, N)$, respectively, describe the time indices of those points of the trajectory which are not contained in a $\rho(P)$ or $\sigma(P)$-neighbourhood of $x^{e}$, respectively, as illustrated in Figure 4.1. The turnpike property then demands that the number of elements contained in these sets, denoted by $\# \mathcal{Q}(x, P, \infty)$ and $\# \mathcal{Q}(x, P, N)$, respectively, is bounded by $P$ while $\rho(P) \rightarrow 0$ and $\sigma(P) \rightarrow 0$ as $P \rightarrow \infty$.

Moreover, for our approach to work we need a regularity condition on the optimal value functions, in the sense that these functions are (approximately) continuous at the equilibrium $\left(x^{e}, u^{e}\right)$ uniformly in $N$. This is Part (iii) of the following assumption.

Assumption 4.1 There is an equilibrium $\left(x^{e}, u^{e}\right) \in \mathbb{Y}$, i.e., a state-control pair with $f\left(x^{e}, u^{e}\right)=x^{e}$, satisfying the following properties.

(i) The infinite horizon optimal control problem for stage cost $\ell(x, u)-\ell\left(x^{e}, u^{e}\right)$ has the turnpike property at $x^{e}$ in the following sense: there exists $\rho \in \mathcal{L}$ 


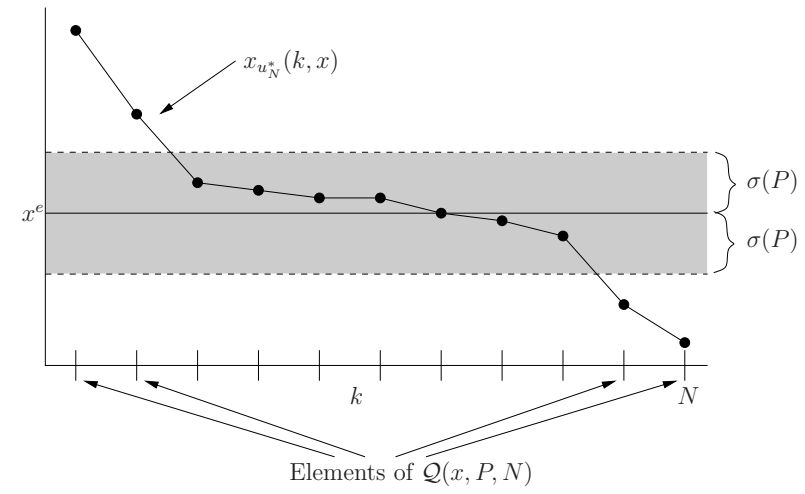

Fig. 4.1 Illustration of the definition of $\mathcal{Q}(x, P, N)$

such that for each optimal trajectory $x_{u_{\infty}^{*}}(k, x), x \in \mathbb{X}$ and all $P \in \mathbb{N}$ there is a set $\mathcal{Q}(x, P, \infty) \subseteq \mathbb{N}_{0}$ with $\# \mathcal{Q}(x, P, \infty) \leq P$ and

$$
\left\|x_{u_{\infty}^{*}}(k, x)-x^{e}\right\| \leq \rho(P) \quad \text { for all } k \in \mathbb{N}_{0} \text { with } k \notin \mathcal{Q}(x, P, \infty) .
$$

(ii) The finite horizon optimal control problems have the turnpike property at $x^{e}$ in the following sense: there exists $\sigma \in \mathcal{L}$ such that for each optimal trajectory $x_{u_{N}^{*}}(k, x), x \in \mathbb{X}$ and all $N, P \in \mathbb{N}$ there is a set $\mathcal{Q}(x, P, N) \subseteq$ $\{0, \ldots, N\}$ with $\# \mathcal{Q}(x, P, N) \leq P$ and

$$
\left\|x_{u_{N}^{*}}(k, x)-x^{e}\right\| \leq \sigma(P) \quad \text { for all } k \in\{0, \ldots, N\} \text { with } k \notin \mathcal{Q}(x, P, N) .
$$

(iii) The optimal value functions $V_{N}$ and $V_{\infty}$ are (approximately) continuous at $x^{e}$ in the following uniform way: there is an open ball $\mathcal{B}_{\varepsilon}\left(x^{e}\right), \varepsilon>0$, around $x^{e}$ and $\eta \in \mathcal{K}_{\infty}, \omega \in \mathcal{L}$ such that for all $x \in \mathcal{B}_{\varepsilon}\left(x^{e}\right) \cap \mathbb{X}$ and all $N \in \mathbb{N} \cup\{\infty\}$ the inequality

$$
\left|V_{N}(x)-V_{N}\left(x^{e}\right)\right| \leq \eta\left(\left\|x-x^{e}\right\|\right)+\omega(N)
$$

holds, where we use the convention $\omega(\infty)=0$.

The motivation for considering the shifted stage cost $\ell(x, u)-\ell\left(x^{e}, u^{e}\right)$ in Part (i) of this assumption is as follows: Since the turnpike property demands that the optimal trajectory stays most of the time near $x^{e}$, under standard continuity assumptions we can expect that $J_{K}\left(x, u_{\infty}^{*}\right)$ takes values near $K \ell\left(x^{e}, u^{e}\right)$. Thus, for $V_{\infty}(x)$ to be finite (which we assumed in Section 2), $\ell\left(x^{e}, u^{e}\right)=0$ is a reasonable assumption which is clearly satisfied for the stage cost $\ell(x, u)-\ell\left(x^{e}, u^{e}\right)$. In contrast to this, for the finite horizon problem the optimal trajectories for stage cost $\ell(x, u)$ and $\ell(x, u)-\ell\left(x^{e}, u^{e}\right)$ coincide because the addition of a constant to $\ell$ only adds a term to the optimisation criterion which is independent of $u$. Hence, in Part (ii) there is no need to consider a shifted stage cost. 
We note that the fact that the bounds in (i) and (ii) are assumed to be independent of $x$ may be restrictive if $\mathbb{X}$ is unbounded. However, since the interesting dynamics usually occur in a bounded set $\mathbb{X}$, we decided to make this assumption which considerably simplifies the subsequent arguments.

The intuition behind (i) and (ii) is that the solution on infinite or long finite time intervals, respectively, spends most of the time near the equilibrium point $x^{e}$. Figure 4.2 shows numerically computed finite horizon optimal trajectories for varying $N$ for Examples (3.1) and (3.2) which indicate that the systems indeed exhibit the turnpike property, since the number of time instants at which the solutions are outside a neighbourhood of the respective equilibria $x^{e}=0$ and $x^{e} \approx 2.234421144$ is clearly independent of $N$.
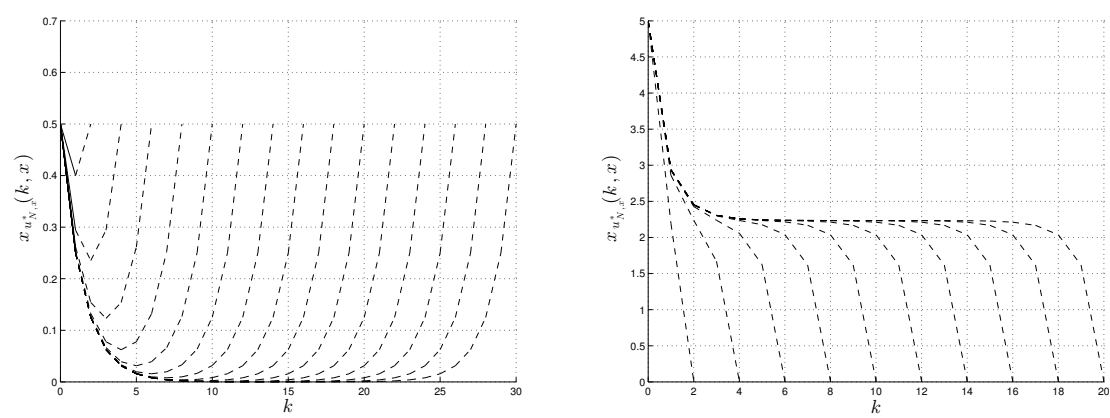

Fig. 4.2 Finite horizon optimal trajectories $x_{u_{N}^{*}}(\cdot, x)$ (dashed) for different optimisation horizons $N=2,4, \ldots, 30$ for Example 3.1 (left) and $N=2,4, \ldots, 20$ for Example 3.2 (right)

Of course, these numerical simulations only provide evidence but not a rigorous proof that the systems indeed have the turnpike property. We will see in the next section how this property can be checked rigorously.

The condition from Assumption 4.1 (iii) is a (rather weak) way of formalising that the optimal solution starting at some point in the neighbourhood of $x^{e}$ is similar to the optimal solution starting at $x^{e}$ itself. Together with Assumption 4.1 (i) and (ii) it implies that for determining the value of an approximately optimal trajectory on a long horizon it suffices to know the value $J_{K}\left(x, u_{N}^{*}\right)$ of an initial piece of the optimal trajectory which ends up near $x^{e}$ and the optimal value in the equlibrium $x^{e}$. This is formalised by the following lemma.

Lemma 4.2 (i) If Assumption 4.1 (i) and (iii) hold, then the equation

$$
V_{\infty}(x)=J_{K}\left(x, u_{\infty}^{*}\right)+V_{\infty}\left(x^{e}\right)+R_{1}(x, K)
$$

holds with $\left|R_{1}(x, K)\right| \leq \eta(\rho(P))$ for all $x \in X$, all sufficiently large $P \in \mathbb{N}$ and all $K \notin \mathcal{Q}(x, P, \infty)$. 
(ii) If Assumption 4.1 (ii) and (iii) hold, then the equation

$$
V_{N}(x)=J_{K}\left(x, u_{N}^{*}\right)+V_{N-K}\left(x^{e}\right)+R_{2}(x, K, N)
$$

holds with $\left|R_{2}(x, K, N)\right| \leq \eta(\sigma(P))+\omega(N-K)$ for all $x \in X$, all $N \in \mathbb{N}$, all sufficiently large $P \in \mathbb{N}$ and all $K \notin \mathcal{Q}(x, P, N)$.

Proof (i) The dynamic programming principle yields

$$
V_{\infty}(x)=J_{K}\left(x, u_{\infty}^{*}\right)+V_{\infty}\left(x_{u_{\infty}^{*}}(K, x)\right) .
$$

Hence, (4.1) holds with $R_{1}(x, K)=V_{\infty}\left(x_{u_{\infty}^{*}}(K, x)\right)-V_{\infty}\left(x^{e}\right)$. Now choose $P \in \mathbb{N}$ sufficiently large that $\rho(P)<\varepsilon$ holds for $\rho$ from Assumption 4.1 (i) and $\varepsilon$ from Assumption 4.1 (iii). Then for $K \in \mathcal{Q}(x, P, \infty)$ we obtain $\left|R_{1}(x, K)\right| \leq \eta\left(\left\|x_{u_{\infty}^{*}}(K, x)-x^{e}\right\|\right) \leq \eta(\rho(K))$ and thus the assertion.

(ii) In the finite horizon case, the dynamic programming principle yields

$$
V_{N}(x)=J_{K}\left(x, u_{N}^{*}\right)+V_{N-K}\left(x_{u_{N}^{*}}(K, x)\right) .
$$

Hence, (4.2) holds with $R_{2}(x, K, N)=V_{N-K}\left(x_{u_{N}^{*}}(K, x)\right)-V_{N-K}\left(x^{e}\right)$. Now choose $P \in \mathbb{N}$ sufficiently large that $\sigma(P)<\varepsilon$ holds for $\sigma$ from Assumption 4.1 (ii) and $\varepsilon$ from Assumption 4.1 (iii). For any $K \in \mathcal{Q}(x, P, N)$ this implies $\left|R_{2}(x, K, N)\right| \leq \eta\left(\left\|x_{u_{N}^{*}}(K, x)-x^{e}\right\|\right)+\omega(N-K) \leq \eta(\sigma(P))+\omega(N-K)$ and thus the assertion.

A consequence of this lemma - and thus of Assumption 4.1 (i)-(iii) - is that the values of the initial pieces of finite and infinite trajectories, respectively, approximately coincide, as the next lemma shows.

Lemma 4.3 If Assumption 4.1 (i)-(iii) holds, then the equation

$$
J_{K}\left(x, u_{\infty}^{*}\right)=J_{K}\left(x, u_{N}^{*}\right)+R_{3}(x, K, N)
$$

holds with $\left|R_{3}(x, K, N)\right| \leq \eta(\rho(P))+\eta(\sigma(P))+2 \omega(N-K)$ for all sufficiently large $P \in \mathbb{N}$, all $x \in \mathbb{X}$ and all $K \in\{0, \ldots, N\} \backslash(\mathcal{Q}(x, P, N) \cup \mathcal{Q}(x, P, \infty))$.

Proof The finite horizon dynamic programming principle implies that $u=$ $u_{N}^{*}$ minimises the expression $J_{K}(x, u)+V_{N-K}\left(x_{u}(K, x)\right)$. Together with the error term $R_{2}$ defined in the proof of Lemma 4.2 (ii) and $\widetilde{R}_{1}(x, K, N)=$ $V_{N-K}\left(x_{u_{\infty}^{*}}(K, x)\right)-V_{N-K}\left(x^{e}\right)$ this yields

$$
\begin{aligned}
& J_{K}\left(x, u_{N}^{*}\right)+V_{N-K}\left(x^{e}\right)=J_{K}\left(x, u_{N}^{*}\right)+V_{N-K}\left(x_{u_{N}^{*}}(K, x)\right)-R_{2}(x, K, N) \\
& \quad \geq J_{K}\left(x, u_{\infty}^{*}\right)+V_{N-K}\left(x_{u_{\infty}^{*}}(K, x)\right)-R_{2}(x, K, N) \\
& \quad=J_{K}\left(x, u_{\infty}^{*}\right)+V_{N-K}\left(x^{e}\right)-R_{2}(x, K, N)+\widetilde{R}_{1}(x, K, N) .
\end{aligned}
$$

Similar to the proof of Lemma 4.2 (ii) one sees that $\left|\widetilde{R}_{1}(x, K, N)\right| \leq \eta(\rho(P))+$ $\omega(N-K)$ for all $K \in \mathcal{Q}(x, P, \infty)$.

Conversely, the infinite horizon dynamic programming principle implies that $u_{\infty}^{*}$ minimises the expression $J_{K}\left(x, u_{\infty}^{*}\right)+V_{\infty}\left(x_{u_{\infty}^{*}}(K, x)\right)$. Using the error 
terms $R_{1}$ from the proof of Lemma $4.2(\mathrm{i})$ and $\widetilde{R}_{2}(x, K, N)=V_{\infty}\left(x_{u_{N}^{*}}(K, x)\right)-$ $V_{\infty}\left(x^{e}\right)$ we obtain

$$
\begin{aligned}
J_{K}\left(x, u_{\infty}^{*}\right)+V_{\infty}\left(x^{e}\right) & =J_{K}\left(x, u_{\infty}^{*}\right)+V_{\infty}\left(x_{u_{\infty}^{*}}(K, x)\right)-R_{1}(x, K) \\
& \geq J_{K}\left(x, u_{N}^{*}\right)+V_{\infty}\left(x_{u_{N}^{*}}(K, x)\right)-R_{1}(x, K) \\
& =J_{K}\left(x, u_{N}^{*}\right)+V_{\infty}\left(x^{e}\right)-R_{1}(x, K)+\widetilde{R}_{2}(x, K, N) .
\end{aligned}
$$

As in the proof of Lemma 4.2 (i) one obtains $\left|\widetilde{R}_{2}(x, K, N)\right| \leq \eta(\sigma(P))$ for all $K \in \mathcal{Q}(x, P, N)$. Together with the estimates for $R_{1}$ and $R_{2}$ from Lemma 4.2 this yields

$$
\begin{aligned}
& \left|R_{3}(x, K, N)\right|=\left|J_{K}\left(x, u_{\infty}^{*}\right)-J_{K}\left(x, u_{N}^{*}\right)\right| \\
& \quad \leq \max \left\{\left|R_{1}(x, K)\right|+\left|\widetilde{R}_{2}(x, K, N)\right|,\left|\widetilde{R}_{1}(x, K, N)\right|+\left|R_{2}(x, K, N)\right|\right\} \\
& \quad \leq \eta(\rho(P))+\eta(\sigma(P))+2 \omega(N-K) .
\end{aligned}
$$

The properties derived in the preceding two lemmas are the key for the first receding horizon approximation result, formulated in the next theorem. For interpreting this result we recall that by the dynamic programming principle (2.6) for each control $u$ and each $M \in \mathbb{N}$ the inequality

$$
J_{M}(x, u) \geq V_{\infty}(x)-V_{\infty}\left(x_{u}(M)\right)
$$

holds. Consequently, a finite horizon control sequence $u$ is part of an infinite horizon optimal control sequence if and only if the inequality

$$
J_{M}(x, u) \leq V_{\infty}(x)-V_{\infty}\left(x_{u}(M)\right)
$$

holds. The following theorem now shows that the RHC closed loop solution satisfies (4.4) up to an error term vanishing as $N \rightarrow \infty$. We remark that for $V_{\infty}$ to be bounded, in the theorem the shifted stage cost $\ell(x, u)-\ell\left(x^{e}, u^{e}\right)$ must be used for the infinite horizon functional, and in order to be consistent it must be used for $J_{M}^{c l}\left(x, \mu_{N}\right)$, too. However, there is no need to use the shifted stage cost in Algorithm 2.1, because the finite horizon optimal trajectories and controls do not change when a constant is added to $\ell$. Hence, the shifting of $\ell$ is only necessary for the analysis of the optimality properties of the RHC solution but not for its computation.

Theorem 4.4 If Assumption 4.1 (i)-(iii) holds and $V_{\infty}$ is bounded on $\mathbb{X}$, then the inequality

$$
J_{M}^{c l}\left(x, \mu_{N}\right) \leq V_{\infty}(x)-V_{\infty}\left(x_{\mu_{N}}(M)\right)+M \delta(N)
$$

holds for all $M \in \mathbb{N}$ and all sufficiently large $N \in \mathbb{N}$ with

$$
\delta(N):=2 \eta(\rho(\lfloor(N-1) / 8\rfloor))+2 \eta(\sigma(\lfloor(N-1) / 8\rfloor))+4 \omega(\lfloor N / 2\rfloor),
$$

where $\lfloor y\rfloor$ denotes the largest integer less or equal $y$ for $y \in \mathbb{R}$. 
Proof Picking $x \in \mathbb{X}$ and abbreviating $x^{+}:=f\left(x, \mu_{N}(x)\right)$, the dynamic programming principle and the definition of $\mu_{N}$ yield

$$
\ell\left(x, \mu_{N}(x)\right)=V_{N}(x)-V_{N-1}\left(x^{+}\right)=J_{K}\left(x, u_{N, x}^{*}\right)-J_{K-1}\left(x^{+}, u_{N-1, x^{+}}^{*}\right),
$$

where the last equality follows from the fact that, by (2.7), the optimal control $u_{N-1, x^{+}}^{*}$ for $x^{+}$coincides with $u_{N}^{*}(\cdot+1)$. Using (4.1) for $N, x$ and $K$ and with $N-1, x^{+}$and $K-1$, respectively, yields

$$
\begin{aligned}
& V_{\infty}(x)-V_{\infty}\left(x^{+}\right) \\
& =J_{K}\left(x, u_{\infty}^{*}\right)+V_{\infty}\left(x^{e}\right)+R_{1}(x, K) \\
& \quad-\quad J_{K-1}\left(x^{+}, u_{\infty}^{*}\right)-V_{\infty}\left(x^{e}\right)-R_{1}\left(x^{+}, K-1\right) \\
& =J_{K}\left(x, u_{\infty}^{*}\right)-J_{K-1}\left(x^{+}, u_{\infty}^{*}\right)+R_{1}(x, K)-R_{1}\left(x^{+}, K-1\right) .
\end{aligned}
$$

Putting the two equations together and using Lemma 4.3 yields

$$
\ell\left(x, \mu_{N}(x)\right)=V_{\infty}(x)-V_{\infty}\left(x^{+}\right)+R_{4}(x, K, N) .
$$

with

$R_{4}(x, K, N)=-R_{3}(x, K, N)+R_{3}\left(x^{+}, K-1, N-1\right)-R_{1}(x, K)+R_{1}\left(x^{+}, K-1\right)$.

From Lemma 4.2(i) and 4.3 we obtain the bound

$$
\left|R_{4}(x, K, N)\right| \leq 2 \eta(\rho(P))+2 \eta(\sigma(P))+4 \omega(N-K)
$$

provided $P \in \mathbb{N}$ is sufficiently large and we choose $K \in\{1, \ldots, N\}$ with $K \notin \mathcal{Q}(x, P, N) \cup \mathcal{Q}(x, P, \infty)$ and $K-1 \notin \mathcal{Q}\left(x^{+}, P, N-1\right) \cup \mathcal{Q}\left(x^{+}, P, \infty\right)$. Since each of the four $\mathcal{Q}$ sets contains at most $P$ elements, their union contains at most $4 P$ elements and hence if $N>8 P$ then there is at least one such $K$ with $K \leq N / 2$.

Thus, choosing $P=\lfloor(N-1) / 8\rfloor$ yields the existence of $K \leq N / 2$ such that $\left|R_{4}(x, K, N)\right| \leq 2 \eta(\rho(\lfloor(N-1) / 8\rfloor))+2 \eta(\sigma(\lfloor(N-1) / 8\rfloor))+4 \omega(\lfloor N / 2\rfloor)=\delta(N)$.

Applying (4.6), (4.7) for $x=x_{\mu_{N}}(k, x), k=0, \ldots, M-1$, we can conclude

$$
\begin{aligned}
J_{M}^{c l}\left(x, \mu_{N}\right) & =\sum_{k=0}^{M-1} \ell\left(x_{\mu_{N}}(k, x), \mu_{N}\left(x_{\mu_{N}}(k, x)\right)\right) \\
& \leq \sum_{k=0}^{M-1}\left(V_{\infty}\left(x_{\mu_{N}}(k, x)\right)-V_{\infty}\left(x_{\mu_{N}}(k+1, x)\right)+\delta(N)\right) \\
& \leq V_{\infty}(x)-V_{\infty}\left(x_{\mu_{N}}(M)\right)+M \delta(N) .
\end{aligned}
$$

This proves the claim.

The following remark collects a couple of comments and extensions related to Theorem 4.4. 
Remark 4.5 (i) Rewriting (4.5) as

$$
J_{M}^{c l}\left(x, \mu_{N}\right)+V_{\infty}\left(x_{\mu_{N}}(M)\right) \leq V_{\infty}(x)+M \delta(N),
$$

we can interpret this inequality as follows: if we control using the RHC feedback law $\mu_{N}$ until time $M$ and then continue controlling using the infinite horizon optimal control, then the resulting overall trajectory will be infinite horizon optimal except for the error term $M \delta(N)$. This means that the RHC trajectory is the initial piece of an approximately optimal infinite horizon trajectory.

(ii) In general, we cannot avoid the multiplicative factor $M$ in the error estimate, see Example 6.7. This also means that in general the limit superior of the performance measure $\lim \sup _{M \rightarrow \infty} J_{M}^{c l}\left(x, \mu_{N}\right)$ may not be finite. However, this does not mean that the performance on arbitrary large horizons deteriorates completely. Assuming that $V_{\infty}$ is bounded along $x_{\mu_{N}}(M)$ (which is true, e.g., if $x_{\mu_{N}}(k)$ ends up in a neighbourhood of $x^{e}$, cf. the subsequent item (iii)) we still obtain the estimate

$$
\limsup _{M \rightarrow \infty} \frac{1}{M} J_{M}^{c l}\left(x, \mu_{N}\right) \leq \delta(N)
$$

on the long time average performance.

(iii) Under additional conditions (for details see Remark 5.8) one can show that $\left\|x_{\mu_{N}}(k)-x^{e}\right\| \leq \gamma(N)$ for a function $\gamma \in \mathcal{L}$ and all sufficiently large $k \in \mathbb{N}$, i.e., that the RHC trajectory converges to a ball with radius $\gamma(N)$ around $x^{e}$. Assuming this inequality is true for some $k=M \in \mathbb{N}$, one can conclude that

$$
J_{M}^{c l}\left(x, \mu_{N}\right) \leq J_{M}(x, u)+M \delta(N)+2 \eta(\gamma(M))
$$

holds for all control sequences $u$ satisfying $\left\|x_{u}(K)-x^{e}\right\| \leq \gamma(N)$ and $\eta$ from Assumption (4.1) (iii). This means that up to the error term $M \delta(N)+$ $2 \eta(\gamma(M))$, RHC generates an optimal trajectory from the initial condition $x$ to the ball with radius $\gamma(N)$ around $x^{e}$. In order to show (4.8), assume this inequality does not hold. This means that we can find a control $\tilde{u}$ with

$$
J_{M}^{c l}\left(x, \mu_{N}\right)>J_{M}(x, \tilde{u})+K \delta(N)+2 \eta(\gamma(M))
$$

and $\left\|x_{\tilde{u}}(M)-x^{e}\right\| \leq \gamma(N)$. Using that Assumption 4.1 (iii) implies $\mid V_{\infty}\left(x_{\tilde{u}}(M)\right)$ $V_{\infty}\left(x^{e}\right) \mid \leq \eta(\gamma(N))$ and $\left|V_{\infty}\left(x_{\mu_{N}}(M)\right)-V_{\infty}\left(x^{e}\right)\right| \leq \eta(\gamma(N))$, we obtain

$$
\begin{aligned}
J_{M}(x, \tilde{u})+V_{\infty}\left(x_{\tilde{u}}(M)\right) & <J_{M}^{c l}\left(x, \mu_{N}\right)+V_{\infty}\left(x_{\tilde{u}}(M)\right)-M \delta(N)-2 \eta(\gamma(M)) \\
& \leq J_{M}^{c l}\left(x, \mu_{N}\right)+V_{\infty}\left(x_{\mu_{N}}(M)\right)-M \delta(N) \\
& \leq V_{\infty}(x),
\end{aligned}
$$

contradicting the dynamic programming principle (2.6).

(iv) In the setting of (iii), the explicit use of $V_{\infty}$ and $u_{\infty}^{*}$ can be avoided in the analysis, at the expense of a more technical proof requiring more involved bookkeeping, see [32], particularly Theorem 4.1 in this reference. This avoids the slightly awkward consideration of $\ell(x, u)-\ell\left(x^{e}, u^{e}\right)$, cf. the discussion before Theorem 4.4 . 
(v) Analogous results can be obtained for discounted optimal control problems [31]. Parts of the results were also carried over to optimal control problems which exhibit an optimal periodic orbit instead of an optimal equilibrium [41].

\section{Alternative sufficient conditions}

The conditions formulated in Assumption 4.1 are difficult to check directly, as they involve the knowledge of optimal trajectories or optimal value functions, respectively. For Assumption 4.1 (i), moreover the correct equilibrium value $\ell\left(x^{e}, u^{e}\right)$ which should be subtracted from $\ell$ needs to be known.

For developing an alternative checkable sufficient condition, the following structural property plays a pivotal role.

Definition 5.1 The optimal control problem is called strictly dissipative at an equilibrium $\left(x^{e}, u^{e}\right)$, if there exists a storage function $\lambda: \mathbb{X} \rightarrow \mathbb{R}$, bounded from below, and a function $\alpha \in \mathcal{K}_{\infty}$ such that the inequality

$$
\lambda(f(x, u)) \leq \lambda(x)+\ell(x, u)-\ell\left(x^{e}, u^{e}\right)-\alpha\left(\left\|x-x^{e}\right\|\right)
$$

holds for all $(x, u) \in \mathbb{Y}$ with $f(x, u) \in \mathbb{X}$.

The concept of strict dissipativity was introduced in mathematical systems theory in $[53,54]$, for its extension to discrete time see [13]. Its importance for receding horizon control was first observed in $[5,6]$. One easily sees that under this condition $\left(x^{e}, u^{e}\right)$ is a global minimum of the optimisation problem

$$
\operatorname{mimimise} \ell(x, u) \quad \text { s.t. }(x, u) \in \mathbb{Y}, f(x, u)=x \text {, }
$$

i.e., $\left(x^{e}, u^{e}\right)$ is a globally optimal equilibrium.

As we will see in the proof of Proposition 5.3, strict dissipativity moreover implies that staying away from the optimal equilibrium for a long time becomes expensive, hence optimal trajectories are forced to move to $x^{e}$, provided moving to $x^{e}$ is not more expensive than staying away. The following definition prevents this situation.

Definition 5.2 An equilibrium $\left(x^{e}, u^{e}\right)$ is called cheaply reachable if there exists $E \in \mathbb{R}$ such that for all $x \in X$ and $N \in \mathbb{N}$ the inequality

$$
V_{N}(x) \leq N \ell\left(x^{e}, u^{e}\right)+E
$$

holds. If $\ell$ is replaced by $\ell(x, u)-\ell\left(x^{e}, u^{e}\right)$, the definition can be extended to $N=\infty$ by requiring

$$
V_{\infty}(x) \leq E
$$

for all $x \in \mathbb{X}$. 
The name "cheaply reachable" is motivated by the fact that Definition 5.2 is satisfied if there are constants $P$ and $\widetilde{E}$ such that for each initial condition $x_{0}$ there is a trajectory from $x_{0}$ to $x^{e}$ in less than $P$ steps and whose cost is bounded by $\widetilde{E}$, i.e., $x^{e}$ can be reached without paying a too high cost. However, the inequality in this definition may also be true in other situations, e.g., when $x^{e}$ is only asymptotically reachable.

Proposition 5.3 If the optimal control problem is strictly dissipative with bounded storage function $\lambda$ at an equilibrium $\left(x^{e}, u^{e}\right)$ and $\left(x^{e}, u^{e}\right)$ is cheaply reachable then the finite horizon turnpike property from Assumption 4.1(ii) holds and if $\ell(x, u)$ is replaced by $\ell(x, u)-\ell\left(x^{e}, u^{e}\right)$ then the infinite horizon turnpike property from Assumption 4.1(i) holds.

Proof First observe that strict dissipativity implies the identity

$$
J_{N}(x, u) \geq \lambda(x(N))-\lambda\left(x_{0}\right)+N \ell\left(x^{e}, u^{e}\right)+\sum_{k=0}^{N-1} \alpha\left(\left\|x_{u}(k)-x^{e}\right\|\right) .
$$

This inequality shows that staying away from $x^{e}$ for a long time indeed becomes expensive, because the sum over $\alpha$ grows unboundedly if the solution stays outside a neighbourhood of $x^{e}$, while all other terms on the right hand side of (5.1) are either bounded or independent of $u$.

We first prove that Assumption 4.1(ii) holds. We prove by contradiction that the assertion holds for

$$
\sigma(P):=\alpha^{-1}\left(\frac{2 M_{\lambda}+E}{P}\right)
$$

where $M_{\lambda}>0$ denotes a bound on $|\lambda|$. To this end, assume that the property from Assumption 4.1(ii) does not hold, i.e., that there are $N \in \mathbb{N}, x \in \mathbb{X}$ and $P \in \mathbb{N}$ such that the number of elements $k \in \mathcal{Q}(x, N, P)$, i.e., the number of those $k \in\{0, \ldots N\}$ which satisfy $\left\|x_{u_{N}^{*}}(k, x)-x^{e}\right\|>\sigma(P)$, is larger than $P$. Using (5.1) with $u=u_{N}^{*}$ and taking only those elements in the sum into account for which $\left\|x_{u_{N}^{*}}(k, x)-x^{e}\right\|>\sigma(P)$ holds, this implies

$$
\begin{aligned}
J_{N}\left(x, u_{N}^{*}\right) & >-2 M_{\lambda}+N \ell\left(x^{e}, u^{e}\right)+P \alpha(\sigma(P)) \\
& =-2 M_{\lambda}+N \ell\left(x^{e}, u^{e}\right)+2 M_{\lambda}+E=N \ell\left(x^{e}, u^{e}\right)+E .
\end{aligned}
$$

This inequality, however, contradicts the assumed cheap reachability since $J_{N}\left(x, u_{N}^{*}\right)=V_{N}(x) \leq N \ell\left(x^{e}, u^{e}\right)+E$.

The validity of Assumption 4.1(i) is proved similarly, using the same $\sigma$ as above and replacing $\ell(x, u)$ by $\ell(x, u)-\ell\left(x^{e}, u^{e}\right)$, after which $\ell\left(x^{e}, u^{e}\right)=0$ holds. Cheap reachability then implies $J\left(x, u_{\infty}^{*}\right)=V_{\infty}(x) \leq E$. In order to obtain a contradiction, assume that the number of elements $k \in \mathcal{Q}(x, N, P)$ is larger than $P$. Let $M$ be larger than the $(P+1)$ st time index in $\mathcal{Q}(x, N, P)$. Then we obtain

$$
\begin{aligned}
J_{M}\left(x, u_{\infty}^{*}\right) & \geq-2 M_{\lambda}+(P+1) \alpha(\sigma(P)) \\
& =-2 M_{\lambda}+2 M_{\lambda}+E+\alpha(\sigma(P))=E+\alpha(\sigma(P))
\end{aligned}
$$


implying

$$
J_{\infty}\left(x, u_{\infty}^{*}\right)=\lim _{M \rightarrow \infty} J_{M}\left(x, u_{\infty}^{*}\right) \geq E+\alpha(\sigma(P))>E
$$

again contradicting the assumed cheap reachability.

We note that the assumptions of Proposition 5.3 imply that $V_{\infty}(x)$ is finite for all $x \in \mathbb{X}$ and the shifted stage cost $\ell(x, u)-\ell\left(x^{e}, u^{e}\right)$, because strict dissipativity with bounded storage function implies $V_{\infty}(x) \geq-2 M_{\lambda}$ while cheap reachability implies $V_{\infty}(x) \leq E$.

As a result of Proposition 5.3, dissipativity can be used as a checkable condition for the turnpike property. In this context it should be noted that using a generalisation of the turnpike property from Assumption 4.1(ii), a converse to Proposition 5.3 can be proved under suitable structural conditions on the system. This means that using strict dissipativity as a checkable condition for the turnpike property is often not overly conservative. For details we refer to [26].

Example 5.4 The optimal control problems in Examples 3.1-3.3 are all strictly dissipative. Indeed, for the first two examples the sufficient conditions from [16, Proposition 4.5] and [16, Proposition 4.3], respectively, are satisfied. This yields strict dissipativity with storage function $\lambda(x)=-x^{2} / 2$ for Example 3.1 and with storage function $\lambda(x)=p^{e}\left(x-x^{e}\right)$ for $p^{e}=\frac{\alpha-1}{\alpha A}$ and $x^{e}=\frac{1}{\frac{1}{\alpha-1}}$ for Example 3.2. In Example 3.3, strict dissipativity of $\left(x^{e}, u^{e}\right)=\left(y_{r e f}, u_{r e f}\right)$ immediately follows for $\lambda \equiv 0$.

Moreover, all examples satisfy the cheap control property. For the first two examples this is easily verified, because for each $x \in \mathbb{X}$ the system can be controlled to the respective $x^{e}$ in one step with uniformly bounded cost. For the Fokker-Planck equation from Example 3.3 the verification is somewhat more involved; we will come back to this in Example 7.5.

In summary, Proposition 5.3 implies that all examples from Section 3 have the turnpike property.

Likewise - but with a somewhat more technically involved proof - one can show that strict dissipativity and cheap reachability together with local controllability in a neighborhood of $x^{e}$ implies the uniform continuity condition from Assumption 4.1(iii). Here, local controllability is defined in the following way.

Definition 5.5 We say that the system is locally controllable at $x^{e}$, if there exists a neighbourhood $\mathcal{N}$ of $x^{e}$, a time $d \in \mathbb{N}$ and $\gamma_{x}, \gamma_{u}, \gamma_{c} \in \mathcal{K}_{\infty}$ such that for any two points $x_{0}, x_{1} \in \mathcal{N}$ there exists $u \in \mathbb{U}^{d}\left(x_{0}\right)$ satisfying $x_{u}\left(d, x_{0}\right)=$ $x_{1}$ and the estimates $\left\|x_{u}\left(k, x_{0}\right)-x^{e}\right\| \leq \gamma_{x}(\delta),\left\|u(k)-u^{e}\right\| \leq \gamma_{u}(\delta)$ and $\left|\ell\left(x_{u}\left(k, x_{0}\right), u(k)\right)-\ell\left(x^{e}, u^{e}\right)\right| \leq \gamma_{c}(\delta)$ for all $k=0, \ldots, d-1$, where $\delta:=$ $\max \left\{\left\|x_{0}-x^{e}\right\|,\left\|x_{1}-x^{e}\right\|\right\}$.

Proposition 5.6 If the optimal control problem is strictly dissipative with bounded storage function $\lambda$ at an equilibrium $\left(x^{e}, u^{e}\right)$ and the system is locally controllable at $x^{e}$, then Assumption 4.1(iii) holds. 
Idea of Proof The first step of the proof consists in observing that optimal solutions which start close to $x^{e}$ stay in a ball with radius $\tilde{\eta}\left(\left\|x_{0}-x^{e}\right\|\right)+\tilde{\omega}(N)$, $\tilde{\eta} \in \mathcal{K}, \tilde{\omega} \in \mathcal{L}$, around $x^{e}$ for a certain number of steps. This is because strict dissipativity implies that moving away from $x^{e}$ and then returning is more expensive than staying near $x^{e}$, which is feasible due to the controllability assumption. Using the controllability property once more, this allows the exchange of the initial pieces of two optimal trajectories starting in $x_{0} \in \mathcal{N}$ and $x^{e}$, respectively, without changing their value by more than an amount which is proportional to $\eta\left(\left\|x_{0}-x^{e}\right\|\right)+\tilde{\omega}(N)$. This implies the desired continuity property.

A detailed proof (even for the more general case where $x^{e}$ is replaced by a periodic orbit) can be found in [41]. This more detailed proof was derived from a similar proof in [25, Section 6], which uses a slightly stronger controllability assumption in order to avoid the $\omega(N-K)$-term in Assumption 4.1(iii).

Example 5.7 It is easy to check directly that the systems in the Examples 3.1 and 3.2 are locally controllable, hence Proposition 5.6 applies.

The Fokker-Planck control system from Example 3.3, however, is not locally controllable, since the space of control functions we are considering (i.e., those which are constant in $x$ ) is simply too small in order to control the system from any state to any other in a neighbourhood $\mathcal{N}$ of $y_{\text {ref }}$. However, the continuity property from Assumption 4.1(iii) nevertheless holds, as we will see in Example 7.5.

Remark 5.8 Strict dissipativity, cheap reachability and local controllability also allow to prove that $x^{e}$ is a practically globally asymptotically stable equilibrium for the RHC solutions, cf. [32]. This in particular means that the RHC solutions converge to a neighbourhood of $x^{e}$ which shrinks down to $x^{e}$ as $N \rightarrow \infty$, cf. also Remark 4.5(ii)-(iv).

We end this section by remarking that under additional conditions exponential decrease of the error terms depending on $N$ can be established. The respective results can be found in [32] and rely on an exponential turnpike property established in [16]. Moreover, parts of the results in this section have also been established for continuous time receding horizon formulations, see $[20,19]$.

\section{Terminal conditions}

A classical approach in receding horizon control is to compensate for the truncation of the optimisation horizon by adding a terminal cost and/or a terminal constraint. For the problem considered in the previous sections, this approach was developed in $[5,6]$, extending earlier work for positive definite stage cost, see, e.g., $[15,38]$.

For this approach, we specify a terminal cost $V_{0}: \mathbb{X}_{0} \rightarrow \mathbb{R}$. Since $V_{0}$ is typically defined only on a subset $\mathbb{X}_{0}$ of $\mathbb{X}$, the so-called terminal constrained 
set, we need to make sure that we optimise only over trajectories which end up in $\mathbb{X}_{0}$. To this end, for each $N \in \mathbb{N}$ and $x \in \mathbb{X}$ we define the set of controls

$$
\mathbb{U}_{\mathbb{X}_{0}}^{N}(x):=\left\{u \in \mathbb{U}^{N}(x) \mid x_{u}(N, x) \in \mathbb{X}_{0}\right\}
$$

which steer the initial condition into $X_{0}$ after $N$ steps.

The terminal conditioned finite horizon cost functional is then defined as

$$
J_{N}^{t c}(x, u):=\sum_{k=0}^{N-1} \ell\left(x_{u}(k, x), u(k)\right)+V_{0}\left(x_{u}(N, x)\right),
$$

the corresponding optimal control problem reads

$$
\underset{u \in \mathbb{U}_{\mathbb{X}_{0}}^{N}(x)}{\operatorname{minimise}} J_{N}^{t c}(x, u)
$$

and the optimal value function is

$$
V_{N}^{t c}(x):=\inf _{u \in \mathbb{U}_{\mathbb{X}_{0}}^{N}(x)} J_{N}(x, u) .
$$

Terminal conditioned receding horizon control now refers to the case where the optimal control problem (6.2) instead of (2.4) is used in Step 2 of the receding horizon Algorithm 2.1. We denote the resulting RHC feedback law by $\mu_{N}^{t c}$ and remark that $\mu_{N}^{t c}$ is defined only on the feasible set $\mathbb{X}_{N}:=\left\{x \in X \mid \mathbb{U}_{\mathbb{X}_{0}}^{N}(x) \neq \emptyset\right\}$, which may constitute a serious practical limitation for this approach.

The crucial assumption on $\mathbb{X}_{0}$ and $V_{0}$ introduced in $[5,6]$ is the following.

Assumption 6.1 There exists an equilibrium $\left(x^{e}, u^{e}\right)$ with $x^{e} \in \mathbb{X}_{0}$ with the following property: for each $x \in \mathbb{X}_{0}$ there exists $u_{x} \in \mathbb{U}(x)$ such that $f(x, u) \in$ $\mathbb{X}_{0}$ and the inequality

$$
V_{0}\left(f\left(x, u_{x}\right)\right) \leq V_{0}(x)-\ell\left(x, u_{x}\right)+\ell\left(x^{e}, u^{e}\right)
$$

holds.

A standard argument (see, e.g., [38]) shows that under Assumption 6.1 $x \in \mathbb{X}_{N}$ implies $f\left(x, \mu_{N}(x)\right) \in \mathbb{X}_{N}$. This property is called recursive feasibility of $\mathbb{X}_{N}$ and guarantees that $x_{\mu_{N}}(k, x) \in \mathbb{X}_{N}$ holds for all $k \in \mathbb{N}$ if (and only if) $x \in \mathbb{X}_{N}$. The simplest choice of $\mathbb{X}_{0}$ and $V_{0}$ satisfying Assumption 6.1 is $\mathbb{X}_{0}=$ $\left\{x^{e}\right\}$ and $V_{0} \equiv 0$. However, the resulting "endpoint constraint" $x_{N}(N, x)=x^{e}$ may lead to problems in the numerical optimisation routine when computing $u_{N}^{*}$, which is why this simple choice is often not preferred from a numerical point of view.

The key advantage of incorporating $\mathbb{X}_{0}$ and $V_{0}$ satisfying Assumption 6.1 into the optimisation lies in the following lemma.

Lemma 6.2 The terminal conditioned optimal value function satisfies the inequality

$$
V_{N}^{t c}\left(f\left(x, \mu_{N}^{t c}(x)\right)\right) \leq V_{N}^{t c}(x)-\ell\left(x, \mu_{N}^{t c}(x)\right)-\ell\left(x^{e}, u^{e}\right)
$$

for all $N \in \mathbb{N}$ and $x \in \mathbb{X}_{N}$. 
Proof Consider the optimal control $u_{N}^{*, t c}$ for initial value $x$ and abbreviate $x^{*}(k)=x_{u_{N}^{*, t c}}(k, x)$. Note that $\mu_{N}^{t c}(x)=u_{N}^{*, t c}(0)$ and $f\left(x, \mu_{N}^{t c}(x)\right)=x^{*}(1)$. Define the control $u^{+}(k):=u_{N}^{*, t c}(k+1)$ for $k=0, \ldots, N-2$ and $u^{+}(N-$ 1) $:=u_{x}$ with $u_{x}$ from Assumption 6.1 for $x=x^{*}(N)$. Abbreviate $x^{+}(k)=$ $x_{u^{+}}\left(k, x^{*}(1)\right)$. These definitions yield

$$
\begin{aligned}
V_{N}^{t c}\left(f\left(x, \mu_{N}^{t c}(x)\right)\right) \leq & J_{N}^{t c}\left(x^{+}(0), u^{+}\right) \\
\leq & V_{N}^{t c}(x)-\ell\left(x, \mu_{N}^{t c}(x)\right) \\
& +\ell\left(x^{+}(N-1), u^{+}(N-1)\right)-V_{0}\left(x^{*}(N)\right)+V_{0}\left(x^{+}(N)\right) \\
= & V_{N}^{t c}(x)-\ell\left(x, \mu_{N}^{t c}(x)\right) \\
& \underbrace{+\ell\left(x^{*}(N), u_{x}\right)-V_{0}\left(x^{*}(N)\right)+V_{0}\left(f\left(x^{*}(N), u_{x}\right)\right)}_{\leq \ell\left(x^{e}, u^{e}\right) \text { by }(6.4)} \\
\leq & V_{N}^{t c}(x)-\ell\left(x, \mu_{N}^{t c}(x)\right)+\ell\left(x^{e}, u^{e}\right),
\end{aligned}
$$

which shows the claim.

When $\ell(x, u)$ is replaced by $\ell(x, u)-\ell\left(x^{e}, u^{e}\right)$, this lemma yields the inequality

$$
\ell\left(x, \mu_{N}^{t c}(x)\right) \leq V_{N}^{t c}(x)-V_{N}^{t c}\left(f\left(x, \mu_{N}^{t c}(x)\right)\right) .
$$

Summing this along the receding horizon trajectory yields

$$
J_{M}^{c l}\left(x, \mu_{N}^{t c}\right) \leq V_{N}^{t c}(x)-V_{N}^{t c}\left(x_{\mu_{N}^{t c}}(M, x)\right) .
$$

As we will see below, under suitable conditions the terms $V_{N}^{t c}$ in the difference on the right hand side of $(6.5)$ can be replaced by $V_{\infty}$ without changing the value of the difference too much. Thus, compared to (4.6), we directly obtain an estimate for $J_{M}^{c l}\left(x, \mu_{N}^{t c}\right)$ instead of an estimate for the single $\ell$. This will allow us to avoid summing up the error term as in the final sum in the proof of Theorem 4.4 and thus enable us to obtain an error term which is independent of $M$ in Theorem 6.6, below.

The following results are analogous to those developed in [27], however, while in this reference as well as in $[5,6]$ the resulting RHC scheme is investigated under the strict dissipativity assumption from Definition 5.1, here we show that we can also obtain an approximation result under an appropriately extended Assumption 4.1. The extension consists of assuming the properties from Assumption 4.1 also for the finite horizon problem with terminal conditions, as formalised in the following assumption.

Assumption 6.3 For the equilibrium $\left(x^{e}, u^{e}\right) \in \mathbb{Y}$ from Assumption 4.1 the following additional conditions hold.

(i) The finite horizon problems with terminal condition have the turnpike property at $x^{e}$ in the following sense: there exists $\sigma^{t c} \in \mathcal{L}$ such that for each optimal trajectory $x_{u_{N}^{*, t c}}(k, x), x \in X$ and all $N, P \in \mathbb{N}$ there is a set $\mathcal{Q}^{t c}(x, P, N) \subseteq\{0, \ldots, N\}$ with $\# \mathcal{Q}^{t c}(x, P, N) \leq P$ and $\left\|x_{u_{N}^{*, t c}}(k, x)-x^{e}\right\| \leq \sigma^{t c}(P) \quad$ for all $k \in\{0, \ldots, N\}$ with $k \notin \mathcal{Q}^{t c}(x, P, N)$. 
(ii) The optimal value functions $V_{N}^{t c}$ are continuous at $x^{e}$ in the following uniform way: there is an open ball $\mathcal{B}_{\varepsilon}\left(x^{e}\right), \varepsilon>0$, around $x^{e}$ and $\eta^{t c} \in \mathcal{K}_{\infty}$, $\omega^{t c} \in \mathcal{L}$ such that for all $x \in \mathcal{B}_{\varepsilon}\left(x^{e}\right)$ and all $N \in \mathbb{N} \cup\{\infty\}$ the inequality

$$
\left|V_{N}^{t c}(x)-V_{N}^{t c}\left(x^{e}\right)\right| \leq \eta^{t c}\left(\left\|x-x^{e}\right\|\right)+\omega^{t c}(N)
$$

holds.

Remark 6.4 By a straightforward adaptation of the arguments from Section 5, one sees that Assumption 6.3 holds if the problem is strictly dissipative and satisfies the reachability and controllability properties from Definitions 5.2 and 5.5 .

The following lemma adapts the technical results from Section 4 to the terminal conditioned case.

Lemma 6.5 (i) If Assumption 6.3 (i), (ii) hold, then the equation

$$
V_{N}^{t c}(x)=J_{K}\left(x, u_{N}^{*, t c}\right)+V_{N-K}^{t c}\left(x^{e}\right)+R_{2}^{t c}(x, K, N)
$$

holds with $\left|R_{2}^{t c}(x, K, N)\right| \leq \eta^{t c}\left(\sigma^{t c}(P)\right)+\omega^{t c}(N-K)$ for all $x \in X$, all $N \in \mathbb{N}$, all sufficiently large $P \in \mathbb{N}$ and all $K \in \mathcal{Q}^{t c}(x, P, N)$.

(ii) If Assumption 4.1 (i), (iii) and Assumption 6.3 (i), (ii) hold, then the equation

$$
J_{K}\left(x, u_{\infty}^{*}\right)=J_{K}\left(x, u_{N}^{*, t c}\right)+R_{3}^{t c}(x, K, N)
$$

holds with $\left|R_{3}^{t c}(x, K, N)\right| \leq \eta^{t c}(\rho(P))+\eta\left(\sigma^{t c}(P)\right)+2 \omega^{t c}(N-K)$ for all sufficiently large $P \in \mathbb{N}$, all $x \in X$ and all $K \in\{0, \ldots, N\} \backslash\left(\mathcal{Q}^{t c}(x, P, N) \cup\right.$ $\mathcal{Q}(x, P, \infty))$.

Proof The proofs are identical to Lemma 4.2(ii) and Lemma 4.3.

Now we can state the terminal conditioned counterpart to Theorem 4.4.

Theorem 6.6 If Assumption 4.1 (i), (iii) and Assumption 6.3 (i), (ii) hold and $V_{\infty}$ is bounded on $\mathbb{X}$, then the inequality

$$
J_{M}^{c l}\left(x, \mu_{N}^{t c}\right) \leq V_{\infty}(x)-V_{\infty}\left(x_{\mu_{N}^{t c}}(M)\right)+\delta^{t c}(N)
$$

holds for all $M \in \mathbb{N}$ and all sufficiently large $N \in \mathbb{N}$ with

$$
\begin{aligned}
\delta(N) \leq & 2 \eta(\rho(\lfloor(N-1) / 8\rfloor))+2 \eta^{t c}\left(\sigma^{t c}(\lfloor(N-1) / 8\rfloor)\right) \\
& +2 \eta^{t c}(\rho(\lfloor(N-1) / 8\rfloor))+2 \eta\left(\sigma^{t c}(\lfloor(N-1) / 8\rfloor)\right)+6 \omega^{t c}(N / 2) .
\end{aligned}
$$

Proof We derive the estimate from inequality (6.5). We abbreviate $x^{M}=$ $x_{\mu_{N}^{t c}}(M, x)$, consider the optimal control $u_{N, x^{M}}^{*, t c}$ for the terminal conditioned problem with initial value $x^{M}$ and apply Lemma 6.5(i). Thus we obtain

$$
\begin{aligned}
J_{M}^{c l}\left(x, \mu_{N}^{t c}\right) & \leq V_{N}^{t c}(x)-V_{N}^{t c}\left(x_{\mu_{N}^{t c}}(M, x)\right) \\
& =J_{K}\left(x, u_{N}^{*, t c}\right)-J_{K}\left(x^{M}, u_{N, x^{M}}^{*, t c}\right)+R_{2}^{t c}(x, K, N)-R_{2}^{t c}\left(x^{M}, K, N\right)
\end{aligned}
$$


for all $K \in\{0, \ldots, N\} \backslash\left(\mathcal{Q}^{t c}(x, P, N) \cup \mathcal{Q}^{t c}\left(x^{M}, P, N\right)\right)$. Using Lemma 6.5 (ii) we can further derive

$$
\begin{aligned}
J_{K}\left(x, u_{N}^{*, t c}\right)-J_{K}\left(x^{M}, u_{N, x^{M}}^{*, t c}\right)= & J_{K}\left(x, u_{\infty}^{*}\right)-J_{K}\left(x^{M}, u_{\infty, x^{M}}^{*}\right) \\
& +R_{3}^{t c}(x, K, N)-R_{3}^{t c}\left(x^{M}, K, N\right)
\end{aligned}
$$

for all times $K \in\{0, \ldots, N\} \backslash\left(\mathcal{Q}^{t c}(x, P, N) \cup \mathcal{Q}(x, P, \infty) \cup \mathcal{Q}^{t c}\left(x^{M}, P, N\right) \cup\right.$ $\left.\mathcal{Q}\left(x^{M}, P, \infty\right)\right)$. Finally, using Lemma $4.2(\mathrm{i})$ we obtain

$$
\begin{aligned}
J_{K}\left(x, u_{\infty}^{*}\right)-J_{K}\left(x^{M}, u_{\infty, x^{M}}^{*}\right)= & V_{\infty}(x)-V_{\infty}\left(x^{M}\right) \\
& +R_{1}(x, P, \infty)-R_{1}\left(x^{M}, P, \infty\right)
\end{aligned}
$$

for all $K \in\{0, \ldots, N\} \backslash\left(\mathcal{Q}(x, P, \infty) \cup \mathcal{Q}\left(x^{M}, P, \infty\right)\right)$. Since the union of the four $\mathcal{Q}$-sets involved in these estimates contains at most $4 P$ values, for $N>8 P$ there is at least one $K \leq N / 2$ contained in none of these sets. Thus, setting $P=\lfloor(N-1) / 8\rfloor$ yields the existence of a common $K \leq N / 2$ satisfying all the above equations and the respective inequalities from Lemma 4.2 and 6.5. This yields

$$
\begin{aligned}
J_{M}^{c l}\left(x, \mu_{N}^{t c}\right) \leq & V_{\infty}(x)-V_{\infty}\left(x_{\mu_{N}^{t c}}(M, x)\right) \\
& +\left|R_{1}(x, P, \infty)\right|+\left|R_{1}\left(x^{M}, P, \infty\right)\right| \\
& +\left|R_{2}^{t c}(x, K, N)\right|+\left|R_{2}^{t c}\left(x^{M}, K, N\right)\right| \\
& +\left|R_{3}^{t c}(x, K, N)\right|-\left|R_{3}^{t c}\left(x^{M}, K, N\right)\right| \\
\leq & V_{\infty}(x)-V_{\infty}\left(x_{\mu_{N}^{t c}}(M, x)\right) \\
& +2 \eta(\rho(\lfloor(N-1) / 8\rfloor))+2 \eta^{t c}\left(\sigma^{t c}(\lfloor(N-1) / 8\rfloor)\right)+2 \omega^{t c}(N / 2) \\
& +2 \eta^{t c}(\rho(\lfloor(N-1) / 8\rfloor))+2 \eta\left(\sigma^{t c}(\lfloor(N-1) / 8\rfloor)\right) \\
& +2 \omega^{t c}(N / 2)+2 \omega^{t c}(N / 2)
\end{aligned}
$$

which shows the claim.

In contrast to the corresponding result without terminal conditions from Theorem 4.4, here the error term is independent of $M$.

Example 6.7 We illustrate this fact by means of Example 3.1, here with $\mathbb{Y}=[-2,2] \times[-3,3]$. For initial condition $x=2$, we compare the values $J_{M}^{c l}\left(x, \mu_{N}^{t c}\right)$ for the $M P C$ scheme with terminal constraint set $\mathbb{X}_{0}=\{0\}$ and terminal cost $V_{0}\left(x^{e}\right)=0$ with the values $J_{M}^{c l}\left(x, \mu_{N}\right)$ for the scheme without any terminal constraints and costs. Figure 6.1 shows the respective values $J_{M}^{c l}\left(x, \mu_{N}^{t c}\right)$ and $J_{M}^{c l}\left(x, \mu_{N}\right)$ for fixed $N=5$ and $M=1, \ldots, 25$. One sees that for small $M$ the RHC trajectories obtained without terminal conditions have advantages, but since one of the error terms without terminal constraints grows linearly in $M$, cf. Remark 4.5(ii), for growing $M$ the performance value of the solutions computed with terminal conditions is better and, in fact, converges to $J_{\infty}^{c l}\left(x, \mu_{N}^{t c}\right)$ as $M \rightarrow \infty$, which here is a finite value.

Figure 6.2 shows the respective values $J_{M}^{c l}\left(x, \mu_{N}^{t c}\right)$ and $J_{M}^{c l}\left(x, \mu_{N}\right)$ for fixed $M=20$ and $N=1, \ldots, 10$. Here one sees that in this example the terminal conditions yield significant improvement for small $N$, while for larger $N$ the difference in performance is negligible. 


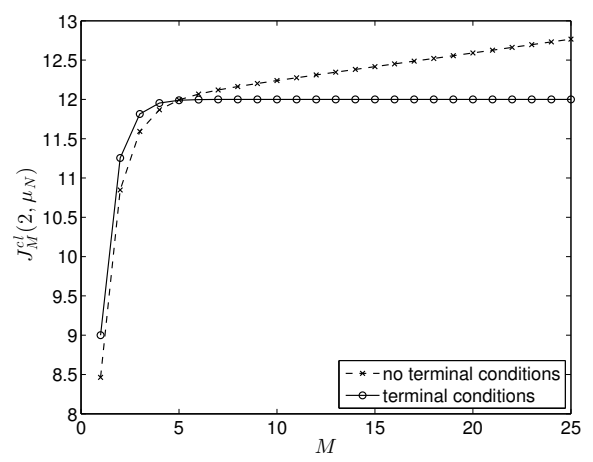

Fig. 6.1 Performance of RHC solution computed with and without terminal conditions for fixed optimization horizon $N=5$ and varying $M=1, \ldots, 25$

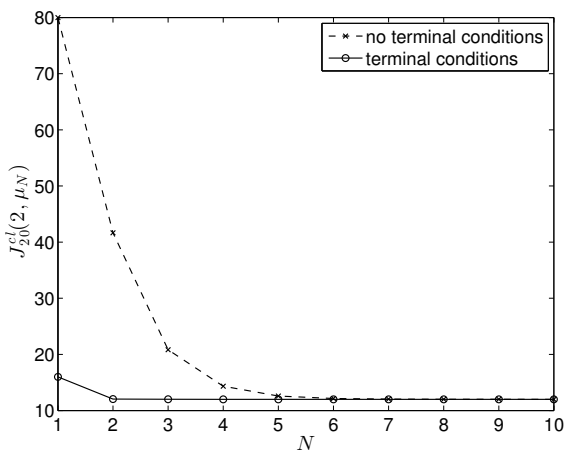

Fig. 6.2 Performance of RHC solutions computed with and without terminal constraints for fixed $M=20$ and varying optimization horizon $N=1, \ldots, 10$

As seen in the example, due to the missing $M$-term in the error estimate, here it does actually make sense to consider the case where $M \rightarrow \infty$. Assuming that $x_{\mu_{N}}(k, x) \rightarrow x^{e}$ as $k \rightarrow \infty$ and $V_{\infty}\left(x^{e}\right)=0$ (both properties follow from strict dissipativity and cheap reachability provided $\ell\left(x^{e}, u^{e}\right)=0$, see $[27])$, under the continuity assumption Assumption 4.1(iii) we obtain that $V_{\infty}\left(x_{\mu_{N}^{t c}}(M, x)\right) \rightarrow 0$ as $M \rightarrow \infty$, implying

$$
J_{\infty}^{c l}\left(x, \mu_{N}^{t c}\right) \leq V_{\infty}(x)+\delta^{t c}(N) .
$$

That is, by incorporating terminal conditions we can even make a statement about the infinite horizon near optimality of the RHC solution.

However, one should take into account that incorporating terminal conditions poses various challenges, from the numerical difficulties of finding a feasible solution under the additional terminal constraints via the fact that the set of feasible initial conditions $\mathbb{X}_{N}$ may be too small, up to the problem of designing $V_{0}$ satisfying (6.4). There are certainly various arguments both for 
and against the use of terminal conditions (see also [37]). These induce a tradeoff between better performance and potential numerical difficulties which each user will need to assess for herself.

\section{Results for tracking problems}

The results from the previous sections can be both simplified and extended if we assume that the stage cost $\ell$ is positive definite w.r.t. $x$ at the equilibrium $\left(x^{e}, u^{e}\right)$, i.e., if there exists $\alpha_{\ell} \in \mathcal{K}_{\infty}$ such that the (in)equalities

$$
\ell\left(x^{e}, u^{e}\right)=0 \quad \text { and } \quad \ell(x, u) \geq \alpha_{\ell}\left(\left\|x-x^{e}\right\|\right) \text { for all }(x, u) \in \mathbb{Y}
$$

hold. We note that the cost function (3.4) for the Fokker-Planck equation is of this type.

In fact, results for this particular setting are much older and widespread in the Receding Horizon/Model Predictive Control literature than for the more general setting discussed before, with the first rigorous performance results in the nonlinear setting dating back at least as far as 1988 [35], see also the historical remarks in the survey paper [38] and the monographs [46,28]. Here we restrict ourselves to the case of $\mathrm{RHC}$ without terminal conditions and refer to the references just cited for variants involving terminal constraints and/or costs.

Optimal control problems with positive definite stage cost usually serve the purpose of asymptotically stabilising the control system at $\left(x^{e}, u^{e}\right)$, in the sense that solutions $x_{\mu_{N}}(k, x)$ stay close to $x^{e}$ if the initial value $x$ is close to $x^{e}$ and that $x_{\mu_{N}}(k, x)$ converges to $x^{e}$ as $k \rightarrow \infty$. In optimal control, stage costs of this type are often referred to as "tracking problems". In control engineering, the term tracking typically refers to the case where $x^{e}$ is time varying, which we will not consider here for simplicity of exposition; we refer to [28] for an extensive treatment of the time varying case.

Besides analysing the performance of the RHC control, in the positive definite case it is therefore necessary to also analyse the asymptotic stability of the controlled system. What at a first glance appears to be an additional complication does not actually pose significant problems, because the two properties, stability and performance, can be addressed at the same time using the following proposition.

Proposition 7.1 Assume there exists $\alpha \in(0,1]$ such that the relaxed dynamic programming inequality

$$
V_{N}\left(f\left(x, \mu_{N}(x)\right)\right) \leq V(x)-\alpha \ell\left(x, \mu_{N}(x)\right)
$$

holds for all $x \in \mathbb{X}$. Then the performance estimate

$$
J_{\infty}^{c l}\left(x, \mu_{N}\right) \leq V_{\infty}(x) / \alpha
$$

holds. If in addition there are $\alpha_{1}, \alpha_{2} \in \mathcal{K}_{\infty}$ such that

$$
\alpha_{1}\left(\left\|x-x^{e}\right\|\right) \leq V_{N}(x) \leq \alpha_{2}\left(\left\|x-x^{e}\right\|\right)
$$


holds for all $x \in \mathbb{X}$, then $x^{e}$ is an asymptotically stable equilibrium for the RHC solutions.

Proof For the first assertion, observe that positive definiteness of $\ell$ implies $V_{N}(x) \geq 0$ and $V_{\infty}(x) \geq V_{N}(x)$ for all $x \in \mathbb{X}$. This implies for each $K \in \mathbb{N}$

$$
\begin{aligned}
\alpha J_{K}^{c l}\left(x, \mu_{N}\right) & =\sum_{k=0}^{K-1} \alpha \ell\left(x_{\mu_{N}}(k, x), \mu_{N}\left(x_{\mu_{N}}(k, x)\right)\right. \\
& \leq \sum_{k=0}^{K-1} V_{N}\left(x_{\mu_{N}}(k, x)-V_{N}\left(x_{\mu_{N}}(k+1, x)\right.\right. \\
& =V_{N}(x)-V_{N}\left(x_{\mu_{N}}(K, x)\right) \leq V_{N}(x) \leq V_{\infty}(x)
\end{aligned}
$$

which shows the claim.

The second assertion follows because under the stated conditions $V_{N}$ is a Lyapunov function for the system, for details we refer to [28, Theorem 4.11].

The performance estimate of Proposition 7.1 was first formulated in this way in [30], motivated by ideas from [36]. However, similar lines of reasoning can be found in earlier literature. It is apparent that it is considerably simpler than the estimates we obtained in the previous sections. Moreover, if we find a way to compute (or at least to estimate) $\alpha$ depending on $N$, then we will be able to give concrete error estimates for fixed $N$ instead of merely asymptotic ones for $N \rightarrow \infty$ as in the previous sections.

Hence, the question now is how to compute $\alpha$ in Proposition 7.1. The assumption we are going to use for this purpose is a bound on the optimal value function of the form

$$
V_{N}(x) \leq \gamma_{N} \ell^{*}(x)
$$

where $\ell^{*}(x):=\inf _{u \in \mathbb{U}(x)} \ell(x, u)$ and $\gamma_{N} \in \mathbb{R}$ with $\gamma_{N} \geq 1$ and $\sup _{N \in \mathbb{N}} \gamma_{N}<\infty$. This condition was independently developed in [51] and [30], however, neither reference presented an optimal estimate of $\alpha$. This was only achieved a little later in $[23,29]$.

We remark that under inequality $(7.2)$ we can apply the theory from Sections 4 and 5 , provided $\ell^{*}(x)$ satisfies the inequality $\ell^{*}(x) \leq \bar{\alpha}_{\ell}\left(\left\|x-x^{e}\right\|\right)$ for some $\bar{\alpha}_{\ell} \in \mathcal{K}$ and is bounded on $\mathbb{X}$ : the positive definiteness of $\ell$ immediately implies that the problem is strictly dissipative (with $\lambda \equiv 0$ ). Boundedness of $\ell^{*}$ together with (7.2) implies the cheap reachability from Definition 5.2. Hence, Proposition 5.3 implies that Assumption 4.1 (i) and (ii) hold. Moreover, the $\bar{\alpha}_{\ell}$-bound on $\ell$ together with (7.2) and the inequality $V_{N}(x) \geq 0$ implies the continuity condition from Assumption 4.1 (iii), even with $\omega \equiv 0$.

However, using (7.1) we can exploit the positive definite structure of the stage cost much more efficiently and obtain the following better estimate. 
Theorem 7.2 Assume (7.2) holds for all $x \in \mathbb{X}$. Then (7.1) holds for all $x \in \mathbb{X}$ and $N \in \mathbb{N}$ with

$$
\alpha=\alpha_{N}=1-\frac{\left(\gamma_{N}-1\right) \prod_{k=2}^{N}\left(\gamma_{k}-1\right)}{\prod_{k=2}^{N} \gamma_{k}-\prod_{k=2}^{N}\left(\gamma_{k}-1\right)}
$$

implying the performance estimate

$$
J_{\infty}^{c l}\left(x, \mu_{N}\right) \leq V_{\infty}(x) / \alpha_{N}
$$

If, moreover, $\sup _{N \in \mathbb{N}} \gamma_{N}<\infty$ holds, then $\alpha_{N} \rightarrow 1$ as $N \rightarrow \infty$.

The proof of this theorem involves the explicit solution of an optimisation problem which computes the worst (i.e., smallest) possible $\alpha$ which is compatible with the bounds (7.2). Unfortunately, the proof is too long to be included in this paper and we refer to [29, Theorem 5.4] or [28, Proposition 6.17]. The same applies to the proof which demonstrates that Theorem 7.2 indeed yields the best possible bound for $\alpha$ (see [23, Theorem 5.3] or [28, Theorem 6.23]).

However, in order to give at least some insight into the mathematical reasoning behind estimating $\alpha$, we present a theorem and proof from [51], which yields a more conservative expression for $\alpha$, but is based on relatively simple arguments which are easy to explain. In order to simplify the presentation, we consider the case where $\gamma_{N}=\gamma$ for all $N$, i.e., where $\gamma_{N}$ does not depend on $N$. We remark that under this assumption $\alpha_{N}$ from Theorem 7.2 simplifies to

$$
\alpha_{N}=1-\frac{(\gamma-1)^{N}}{\gamma^{N-1}-(\gamma-1)^{N-1}} .
$$

Theorem 7.3 Assume (7.2) holds for all $x \in X$ with $\gamma_{N}=\gamma$ independent on $N$. Then (7.1) holds for all $x \in X$ and $N \in \mathbb{N}$ with

$$
\alpha=\tilde{\alpha}_{N}=1-\frac{(\gamma-1)^{N}}{\gamma^{N-2}}
$$

implying the performance estimate

$$
J_{\infty}^{c l}\left(x, \mu_{N}\right) \leq V_{\infty}(x) / \tilde{\alpha}_{N} .
$$

Moreover, $\tilde{\alpha}_{N}$ converges to 1 as $N \rightarrow \infty$.

Proof Consider the optimal trajectory $x_{u_{N}^{*}}(k, x)$ starting at $x$ and abbreviate $\ell_{k}=\ell\left(x_{u_{N}^{*}}(k, x), u_{N}^{*}(k)\right)$. Since by the dynamic programming principle tails of optimal trajectories are again optimal trajectories, see $(2.7)$, for each $p=$ $0, \ldots, N-2$ from $(7.2)$ applied to $x=x_{u_{N}^{*}}(p, x)$ we obtain

$$
\sum_{k=p}^{N-1} \ell_{k}=V_{N-p}\left(x_{u_{N}^{*}}(p, x)\right) \leq \gamma \ell^{*}\left(x_{u_{N}^{*}}(p, x)\right) \leq \gamma \ell_{p}
$$


implying

$$
\sum_{k=p+1}^{N-1} \ell_{k} \leq(\gamma-1) \ell_{p} \quad \text { for all } p=0, \ldots, N-2
$$

which yields

$$
\ell_{p}+\sum_{k=p+1}^{N-1} \ell_{k} \geq \frac{\sum_{k=p+1}^{N-1} \ell_{k}}{\gamma-1}+\sum_{k=p+1}^{N-1} \ell_{k}=\frac{\gamma}{\gamma-1} \sum_{k=p+1}^{N-1} \ell_{k} .
$$

Using this inequality inductively for $p=1, \ldots, N-2$ yields

$$
\sum_{k=1}^{N-1} \ell_{k} \geq\left(\frac{\gamma}{\gamma-1}\right)^{N-2} \ell_{N-1}
$$

Applying (7.3) for $p=0$ we then obtain

$$
(\gamma-1) \ell_{0} \geq \sum_{k=1}^{N-1} \ell_{k} \geq\left(\frac{\gamma}{\gamma-1}\right)^{N-2} \ell_{N-1}
$$

which finally leads to

$$
\ell_{N-1} \leq(\gamma-1)\left(\frac{\gamma-1}{\gamma}\right)^{N-2} \ell_{0}=\gamma\left(\frac{\gamma-1}{\gamma}\right)^{N-1} \ell_{0} .
$$

Moreover, using (7.2) for $x=x_{u_{N}^{*}}(N-1, x)$ and $N=2$ yields

$$
V_{2}\left(x_{u_{N}^{*}}(N-1, x)\right) \leq \gamma \ell_{N-1} .
$$

We denote the optimal control sequence of length 2 corresponding to $V_{2}\left(x_{u_{N}^{*}}(N-\right.$ $1, x))$ by $\tilde{u}_{2}^{*}$. Now, if we use the control sequence $\tilde{u}$ consisting of the control values $\left(u_{N}^{*}(1), \ldots, u_{N}^{*}(N-2), \tilde{u}_{2}^{*}(0), \tilde{u}_{2}^{*}(1)\right)$ we obtain

$$
\begin{aligned}
V_{N}\left(x_{u_{N}^{*}}(1, x)\right) & \leq J_{N}\left(x_{u_{N}^{*}}(1, x), \tilde{u}\right)=\sum_{k=1}^{N-2} \ell_{k}+V_{2}\left(x_{u_{N}^{*}}(N-1, x)\right) \\
& =\sum_{k=1}^{N-1} \ell_{k}-\ell_{N-1}+V_{2}\left(x_{u_{N}^{*}}(N-1, x)\right) \leq \sum_{k=1}^{N-1} \ell_{k}+(\gamma-1) \ell_{N-1} \\
& \leq \sum_{k=1}^{N-1} \ell_{k}+(\gamma-1) \gamma\left(\frac{\gamma-1}{\gamma}\right)^{N-1} \ell_{0} . \\
& =\sum_{k=0}^{N-1} \ell_{k}-\left(1-(\gamma-1) \gamma\left(\frac{\gamma-1}{\gamma}\right)^{N-1}\right) \ell_{0} .
\end{aligned}
$$


Using that by definition of $\mu_{N}$ we have $f\left(x, \mu_{N}(x)\right)=x_{u_{N}^{*}}(1, x)$ and by definition of the $\ell_{k}$ we have $V_{N}(x)=\sum_{k=0}^{N-1} \ell_{k}$, we thus end up with

$$
\begin{aligned}
& V_{N}\left(f\left(x, \mu_{N}(x)\right)\right) \\
& \leq V_{N}(x)-\left(1-\gamma(\gamma-1)\left(\frac{\gamma-1}{\gamma}\right)^{N-1}\right) \ell_{0} \\
& =V_{N}(x)-\left(1-\frac{(\gamma-1)^{N}}{\gamma^{N-2}}\right) \ell_{0}
\end{aligned}
$$

which proves $(7.1)$ with $\alpha=\tilde{\alpha}_{N}=1-(\gamma-1)^{N} / \gamma^{N-2}$.

The convergence $\tilde{\alpha}_{N} \rightarrow 1$ as $N \rightarrow \infty$ follows because $\gamma \geq 1$ yields

$$
\frac{(\gamma-1)^{N}}{\gamma^{N-2}}=\gamma^{2}\left(\frac{\gamma-1}{\gamma}\right)^{N} \rightarrow 0
$$

as $N \rightarrow \infty$.

The difference between the two estimates $\alpha_{N}$ and $\tilde{\alpha}_{N}$ is best seen when we fix $\gamma$ and compute the smallest $N$ for which $\alpha_{N}$ or $\tilde{\alpha}_{N}$ becomes positive, which is the smallest horizon length for which Proposition 7.1 can be applied. Since this is also the smallest horizon length for which the RHC solution is guaranteed to be asymptotically stable, it is called the minimal stabilising horizon. For $\gamma=5$, this number evaluates to $N=9$ for $\alpha_{N}$ and to $N=$ 15 for $\tilde{\alpha}_{N}$. Asymptotically, i.e., for large $\gamma$, one can show that Theorem 7.3 overestimates the minimal stabilising horizon by a factor of $\approx 2$ compared to Theorem 7.2, see [24]. While this clearly shows the conservatism of Theorem 7.3 , the relative simplicity of its proof makes it more suitable for inclusion in this survey paper.

It remains to answer the question of how the $\gamma_{N}$ in (7.2) can be estimated. This question must be dealt with on a case-by-case basis depending on the system under consideration. For instance, in [56] for a model of a differential drive robot the condition $(7.2)$ — in a continuous time version, see below — has been checked by estimating $V_{N}(x)$ via $J_{N}(x, u)$ for suitably constructed control functions $u$. Another approach which has proven successful in several examples is to derive $\gamma_{N}$ from the following exponential controllability assumption w.r.t. $\ell$.

Assumption 7.4 There are $C>0$ and $\sigma \in(0,1)$ with the following property: For each $x \in \mathbb{X}$ there is $u_{x} \in \mathbb{U}^{\infty}(x)$ such that the inequality

$$
\ell\left(x_{u_{x}}(k, x), u_{x}(k)\right) \leq C \sigma^{k} \ell^{*}(x) .
$$

One easily checks that this assumption implies (7.2) with

$$
\gamma_{N}=C \frac{(1-\sigma)^{N}}{1-\sigma}
$$


While it is known that this approach is more conservative than checking (7.2) directly, see [55], for many systems there exist known techniques for checking exponential controllability. In particular, there are quite a number of infinite dimensional control systems governed by PDEs for which this approach is feasible, see, e.g., $[1-4,9]$

Another advantage of working with Assumption 7.4 lies in the fact that the two involved parameters $C$ and $\sigma$ have a rather clear interpretation in the context of computing $\alpha$ in (7.1). In particular, it can be shown that for any fixed value of $\sigma \in(0,1)$ there exists $C_{0}>1$ such that for all $C<C_{0}$ the value $\alpha_{N}$ from Theorem 7.2 is positive for all $N \geq 2$, hence asymptotic stability of the RHC solution follows for all $N \geq 2$, including the shortest meaningful horizon $N=2$, see, e.g., [28, Section 6.6]. We will use this fact in the stability analysis of our Fokker-Planck example.

Example 7.5 For the Fokker-Planck equation corresponding to the OrnsteinUhlenbeck process of Example 3.3, it is shown in [22] that the exponential controllability property from Assumption 7.4 holds for $u$ independent of $x$ whenever $y_{\text {ref }}$ is a Gaussian distribution whose covariance is compatible with the $\sigma_{i j}$ from the Ornstein-Uhlenbeck process and the initial condition is also Gaussian. Moreover, one can show that the constant $C$ in Assumption 7.4 can be chosen arbitrarily close to 1 , such that Theorem 7.2 and Proposition 7.1 yield asymptotic stability of the RHC solution for $N=2$, explaining the behaviour observed in the simulation in Example 3.3. As pointed out in the discussion after equation (7.2), this moreover shows that the continuity property from Assumption 4.1(iii) holds (which, however, is not needed when using Theorem 7.2).

The proof relies on an explicit representation of the solution of the FokkerPlanck equation for constant (in $t$ and $x$ ) control and requires a nontrivial trick: when directly analysing the decay of $\ell(x, u)$ from (3.4), for certain initial conditions one obtains a constant $C \approx 2$ in Assumption 7.4. The trick to arrive at a smaller constant $C$ lies in the observation that one can define an alternative stage cost which produces identical optimal trajectories but for which $C$ is arbitrarily close to 1 . For details we refer to [22].

We end this section by discussing a few extensions and modifications of the central results. Obviously, it may not always be realistic to expect the estimate (7.2) to hold globally, i.e., for all $x \in \mathbb{X}$. This case has been investigated in [11] and further studied in [49].

The discrete time formulation presented here also has a rather mature continuous time counterpart. This was developed in [47] and compared with the discrete time approach in [57]. An infinite dimensional version of the result with application to the Burgers equation has been developed in [9], where again (7.2) is not assumed to hold globally. The latter reference also reports numerical results showing that for the equation under consideration, avoiding stabilising terminal conditions (which for infinite dimensional systems are discussed, e.g., in [33]) significantly increases the performance and reduces the numerical effort. 


\section{Conclusions and future research}

The results in this paper have shown that receding horizon control can be used as a mathematically justified approximate solution technique for infinite horizon optimal control problems. A prerequisite for this is that the optimal control problem has a suitable structure. In this paper, we have in particular shown that the turnpike property from optimal control (plus suitable regularity) provides such a structure. A remarkable feature in this context is that - at least on the case of receding horizon control without terminal conditions - knowledge about the turnpike property is not needed in order to run the algorithm. If the problem has the turnpike property, the algorithm will "automatically" yield an approximately optimal solution.

Future work will focus on the extension of the class of problems for which the method is applicable. On the one hand, this includes extensions of the general assumptions in Section 4 like, e.g., allowing for dynamics and/or stage costs depending on time varying data, which is ubiquitous in practical applications. Here, in particular a suitable time varying extension of the concept of the optimal equilibrium $x^{e}$ must be found. Another extension of this type would be the generalisation of the results from Section 5 to discounted optimal control problems. It is currently still largely open how an appropriate notion of strict dissipativity would have to look like.

On the other hand, it is desirable to verify the abstract conditions from Sections 4 and 5 for more classes of systems. This for instance applies to optimal control problems involving PDEs in which the stage cost is not positive definite, i.e., to which the methods from Section 7 do not apply.

\section{References}

1. N. Altmüller, Model predictive control for partial differential equations. PhD Thesis, Universität Bayreuth, 2014.

2. N. Altmüller And L. Grüne, Distributed and boundary model predictive control for the heat equation, GAMM-Mitt., 35 (2012), pp. 131-145.

3. N. Altmüller AND L. Grüne, A comparative stability analysis of Neumann and Dirichlet boundary MPC for the heat equation, in Proceedings of the 1st IFAC Workshop on Control of Systems Modeled by Partial Differential Equations - CPDE 2013, 2013, pp. $161-166$.

4. N. Altmüller, L. Grüne, and K. Worthmann, Receding horizon optimal control for the wave equation, in Proceedings of the 49th IEEE Conference on Decision and Control - CDC2010, Atlanta, Georgia, 2010, pp. 3427-3432.

5. R. Amrit, J. B. Rawlings, And D. Angeli, Economic optimization using model predictive control with a terminal cost, Annual Rev. Control, 35 (2011), pp. 178-186.

6. D. Angeli, R. Amrit, And J. B. Rawlings, On average performance and stability of economic model predictive control, IEEE Trans. Autom. Control, 57 (2012), pp. 16151626.

7. M. Annunziato And A. Borzì, Optimal control of probability density functions of stochastic processes, Math. Model. Anal., 15 (2010), pp. 393-407.

8. M. Annunziato And A. Borzì, A Fokker-Planck control framework for multidimensional stochastic processes, J. Comput. Appl. Math., 237 (2013), pp. 487-507.

9. B. Azmi AND K. Kunisch, On the stabilizability of the Burgers' equation by receding horizon control. Preprint, TU Graz, 2015. http://www. uni-graz.at/ kunisch/papers/KK_291.pdf. 
10. D. P. Bertsekas, Dynamic Programming and Optimal Control. Vol. 1 and 2., Athena Scientific, Belmont, MA, 1995.

11. A. Boccia, L. Grüne, and K. Worthmann, Stability and feasibility of state constrained MPC without stabilizing terminal constraints, Systems Control Lett., 72 (2014), pp. 14 21.

12. W. A. Brock and L. Mirman, Optimal economic growth and uncertainty: the discounted case, J. Econ. Theory, 4 (1972), pp. 479-513.

13. C. I. Byrnes AND W. Lin, Losslessness, feedback equivalence, and the global stabilization of discrete-time nonlinear systems, IEEE Trans. Automat. Control, 39 (1994), pp. 83-98.

14. D. A. Carlson, A. B. Haurie, and A. Leizarowitz, Infinite horizon optimal control - Deterministic and Stochastic Systems, Springer-Verlag, Berlin, second ed., 1991.

15. H. CHEN AND F. AllGÖWER, A quasi-infinite horizon nonlinear model predictive control scheme with guaranteed stability, Automatica, 34 (1998), pp. 1205-1217.

16. T. Damm, L. Grüne, M. Stieler, and K. Worthmann, An exponential turnpike theorem for dissipative discrete time optimal control problems, SIAM J. Control Optim., 52 (2014), pp. 1935-1957.

17. M. Diehl, R. Amrit, and J. B. Rawlings, A Lyapunov function for economic optimizing model predictive control, IEEE Trans. Autom. Control, 56 (2011), pp. 703-707.

18. R. Dorfman, P. A. Samuelson, and R. M. Solow, Linear Programming and Economic Analysis, Dover Publications, New York, 1987. Reprint of the 1958 original.

19. T. Faulwasser and D. Bonvin, On the design of economic NMPC based on approximate turnpike properties, in Proceedings of the 54rd IEEE Conference on Decision and Control - CDC 2015, 2015, pp. 4964-4970.

20. T. Faulwasser, M. Korda, C. N. Jones, And D. Bonvin, Turnpike and dissipativity properties in dynamic real-time optimization and economic $M P C$, in Proceedings of the 53rd IEEE Conference on Decision and Control — CDC 2014, 2014, pp. 2734-2739.

21. R. Findeisen and F. Allgöwer, An introduction to Nonlinear Model Predictive Control, in 21st Benelux Meeting on Systems and Control, Veldhoven, The Netherlands, 2002, pp. 119-141.

22. A. FLEIG AND L. GRüNE, Estimates on the minimal stabilizing horizon length in Model Predictive Control for the Fokker-Planck equation. Preprint, Universität Bayreuth, 2016. Submitted.

23. L. GRÜNE, Analysis and design of unconstrained nonlinear MPC schemes for finite and infinite dimensional systems, SIAM J. Control Optim., 48 (2009), pp. 1206-1228.

24. L. GRÜNE, NMPC without terminal constraints, in Proceedings of the IFAC Conference on Nonlinear Model Predictive Control - NMPC'12, 2012, pp. 1-13.

25. L. GRÜNE, Economic receding horizon control without terminal constraints, Automatica, 49 (2013), pp. 725-734.

26. L. GRüNe AND M. A. MÜLLER, On the relation between strict dissipativity and the turnpike property, Syst. Contr. Lett., (2016). To appear.

27. L. GRÜNE AND A. PANIN, On non-averaged performance of economic MPC with terminal conditions, in Proceedings of the 54th IEEE Conference on Decision and Control CDC 2015, Osaka, Japan, 2015, pp. 4332-4337.

28. L. Grüne and J. Pannek, Nonlinear Model Predictive Control. Theory and Algorithms, Springer-Verlag, London, 2011.

29. L. Grüne, J. Pannek, M. Seehafer, and K. Worthmann, Analysis of unconstrained nonlinear MPC schemes with time varying control horizon, SIAM J. Control Optim., 48 (2010), pp. 4938-4962.

30. L. GRÜNE AND A. RANTZER, On the infinite horizon performance of receding horizon controllers, IEEE Trans. Automat. Control, 53 (2008), pp. 2100-2111.

31. L. Grüne, W. Semmler, And M. Stieler, Using nonlinear model predictive control for dynamic decision problems in economics, J. Econom. Dynam. Control, 60 (2015), pp. $112-133$.

32. L. Grüne And M. Stieler, Asymptotic stability and transient optimality of economic MPC without terminal conditions, J. Proc. Control, 24 (2014), pp. 1187-1196.

33. K. Ito AND K. Kunisch, Receding horizon optimal control for infinite dimensional systems, ESAIM Control Optim. Calc. Var., 8 (2002), pp. 741-760. A tribute to J. L. Lions. 
34. M. KAgANOVICH, Efficiency of sliding plans in a linear model with time-dependent technology, The Review of Economic Stuidies, 52 (1985), pp. 691-702.

35. S. S. Keerthi And E. G. Gilbert, Optimal infinite horizon feedback laws for a general class of constrained discrete-time systems: stability and moving horizon approximations, J. Optimiz. Theory Appl., 57 (1988), pp. 265-293.

36. B. Lincoln And A. Rantzer, Relaxing dynamic programming, IEEE Trans. Autom. Control, 51 (2006), pp. 1249-1260.

37. D. Q. MAYNe, An apologia for stabilising terminal conditions in model predictive control, Internat. J. Control, 86 (2013), pp. 2090-2095.

38. D. Q. Mayne, J. B. Rawlings, C. V. Rao, and P. O. M. Scokaert, Constrained model predictive control: stability and optimality, Automatica, 36 (2000), pp. 789-814.

39. L. W. MCKenZIE, Optimal economic growth, turnpike theorems and comparative dynamics, in Handbook of Mathematical Economics, Vol. III, vol. 1 of Handbooks in Econom., North-Holland, Amsterdam, 1986, pp. 1281-1355.

40. M. Mohammadi And A. Borzì, Analysis of the Chang-Cooper discretization scheme for a class of Fokker-Planck equations, Journal of Numerical Mathematics, 23 (2015), pp. 271-288.

41. M. A. MÜLler AND L. GRÜNE, Economic model predictive control without terminal constraints for optimal periodic behavior. Preprint, University of Bayreuth, 2015. Submitted.

42. A. Porretta And E. Zuazua, Long time versus steady state optimal control, SIAM J. Control Optim., 51 (2013), pp. 4242-4273.

43. S. Primak, V. Kontorovich, and V. Lyandres, Stochastic methods and their applications to communications, John Wiley \& Sons, Inc., Hoboken, NJ, 2004.

44. A. Propor̆, Application of linear programming methods for the synthesis of automatic sampled-data systems, Avtomat. i Telemeh., 24 (1963), pp. 912-920.

45. P. E. Protter, Stochastic integration and differential equations, vol. 21 of Stochastic Modelling and Applied Probability, Springer-Verlag, Berlin, 2005.

46. J. B. Rawlings and D. Q. Mayne, Model Predictive Control: Theory and Design, Nob Hill Publishing, Madison, Wisconsin, 2009.

47. M. Reble AND F. AllgöWER, Unconstrained model predictive control and suboptimality estimates for nonlinear continuous-time systems, Automatica, 48 (2011), pp. 18121817.

48. H. Risken, The Fokker-Planck equation, vol. 18 of Springer Series in Synergetics, Springer-Verlag, Berlin, second ed., 1989.

49. M. Schulze Darup And M. CAnnon, A missing link between nonlinear MPC schemes with guaranteed stability, in Proceedings of the 54rd IEEE Conference on Decision and Control - CDC 2015, 2015, pp. 4977-4983.

50. E. TRÉLAT AND E. ZuAZuA, The turnpike property in finite-dimensional nonlinear optimal control, J. Differential Equations, 258 (2015), pp. 81-114.

51. S. E. Tuna, M. J. Messina, And A. R. TeEL, Shorter horizons for model predictive control, in Proceedings of the 2006 American Control Conference, Minneapolis, Minnesota, USA, 2006.

52. J. von Neumann, A model of general economic equilibrium, The Review of Economic Studies, 13 (1945), pp. 1-9.

53. J. C. Willems, Dissipative dynamical systems. I. General theory, Arch. Rational Mech. Anal., 45 (1972), pp. 321-351.

54. J. C. Willems, Dissipative dynamical systems. II. Linear systems with quadratic supply rates, Arch. Rational Mech. Anal., 45 (1972), pp. 352-393.

55. K. Worthmann, Stability Analysis of Unconstrained Receding Horizon Control Schemes. PhD Thesis, Universität Bayreuth, 2011.

56. K. Worthmann, M. W. Mehrez, M. Zanon, G. K. I. Mann, R. G. Gosine, and M. DIEHL, Regulation of differential drive robots using continuous time MPC without stabilizing constraints or costs, in Proceedings of the 5th IFAC Conference on Nonlinear Model Predictive Control NMPC 2015, Seville, Spain, vol. 48 of IFAC-PapersOnLine, 2015, pp. 129-135.

57. K. Worthmann, M. Reble, L. Grüne, and F. Allgöwer, The role of sampling for stability and performance in unconstrained nonlinear model predictive control, SIAM J. Cont. Optim., 52 (2014), pp. 581-605. 